\title{
Thrombospondins deployed by thrombopoietic cells determine angiogenic switch and extent of revascularization
}

\author{
Hans-Georg Kopp,, ${ }^{1,2}$ Andrea T. Hooper, ${ }^{1}$ M. Johan Broekman, ${ }^{3}$ Scott T. Avecilla, ${ }^{1}$ \\ Isabelle Petit, ${ }^{1}$ Min Luo, ${ }^{4}$ Till Milde, ${ }^{1}$ Carlos A. Ramos, ${ }^{5}$ Fan Zhang, ${ }^{1}$ Tabitha Kopp, ${ }^{1}$ \\ Paul Bornstein, ${ }^{6}$ David K. Jin, ${ }^{1}$ Aaron J. Marcus,, ${ }^{3,7}$ and Shahin Rafii'

\begin{abstract}
${ }^{1}$ Howard Hughes Medical Institute, Department of Genetic Medicine, Weill Medical College of Cornell University (WMCCU), New York, New York, USA. 'Department of Hematology-Oncology, Eberhard-Karls University, Tubingen, Germany. 'Divisions of Hematology/ Medical Oncology, Medical and Research Service, VA New York Harbor Healthcare System, and Hematology/Medical Oncology, Department of Medicine, WMCCU, New York, New York, USA. ${ }^{4}$ Department of Cell and Developmental Biology, WMCCU, New York, New York, USA. ${ }^{5}$ Department of Medicine, Memorial Sloan-Kettering Cancer Center, New York, New York, USA. ${ }^{6}$ Departments of Biochemistry and Medicine, University of Washington, Seattle, Washington, USA. ${ }^{7}$ Department of Pathology and Laboratory Medicine, WMCCU, New York, New York, USA.
\end{abstract}

\begin{abstract}
Thrombopoietic cells may differentially promote or inhibit tissue vascularization by releasing both pro- and antiangiogenic factors. However, the molecular determinants controlling the angiogenic phenotype of thrombopoietic cells remain unknown. Here, we show that expression and release of thrombospondins (TSPs) by megakaryocytes and platelets function as a major antiangiogenic switch. TSPs inhibited thrombopoiesis, diminished bone marrow microvascular reconstruction following myelosuppression, and limited the extent of revascularization in a model of hind limb ischemia. We demonstrate that thrombopoietic recovery following myelosuppression was significantly enhanced in mice deficient in both TSP1 and TSP2 (TSP-DKO mice) in comparison with WT mice. Megakaryocyte and platelet levels in TSP-DKO mice were rapidly restored, thereby accelerating revascularization of myelosuppressed bone marrow and ischemic hind limbs. In addition, thrombopoietic cells derived from TSP-DKO mice were more effective in supporting neoangiogenesis in Matrigel plugs. The proangiogenic activity of TSP-DKO thrombopoietic cells was mediated through activation of MMP-9 and enhanced release of stromal cell-derived factor 1 . Thus, TSP-deficient thrombopoietic cells function as proangiogenic agents, accelerating hemangiogenesis within the marrow and revascularization of ischemic hind limbs. As such, interference with the release of cellular stores of TSPs may be clinically effective in augmenting neoangiogenesis.
\end{abstract}

\section{Introduction}

In 1878, Theodor Billroth identified viable neoplastic cells in blood clots and introduced the concept that the hemostatic system plays a role in tumor metastasis (1). It is now well known that platelets modulate angiogenesis (2-4). However, since platelets or megakaryocytes elaborate both pro- and antiangiogenic factors, the mechanism by which thrombopoietic cells regulate neoangiogenesis has remained elusive.

Megakaryocytes and platelets produce and release VEGF-A (5) and other proangiogenic cytokines, including bFGF (also known as FGF-2) (6) and MMPs (7), thereby promoting angiogenesis (8). Indeed, increased serum VEGF-A levels in cancer patients originate from platelet activation during serum preparation (9). Furthermore, platelets release proangiogenic lipid mediators, such as sphingosine-1-phosphate (S1P) and lysophosphatidic acid (LPA) $(10,11)$. In vitro, platelets stimulate endothelial cell proliferation and tube formation (12). In vivo models of angiogenesis and tumor progression have established a dose-response relation-

Nonstandard abbreviations used: CA4P, combretastatin A4 phosphate; $\mathrm{Ct}$, threshold cycle; DAB, diaminobenzidine; 5-FU, 5-fluorouracil; 5HT, 5-hydroxytryptamine; PFA, paraformaldehyde; PRP, platelet-rich plasma; $\mathrm{qPCR}$, real-time quantitative PCR; SDF-1, stromal cell-derived factor 1; TSP, thrombospondin; TSP-DKO, TSP double$\mathrm{KO}$ (mice); VE cadherin, vascular endothelial cadherin.

Conflict of interest: The authors have declared that no conflict of interest exists. Citation for this article: J. Clin. Invest. 116:3277-3291 (2006). doi:10.1172/JCI29314. ship between blood platelet levels and sprouting angiogenesis as well as tumor metastasis (4). Moreover, reducing platelet number and/or function has been demonstrated to have antimetastatic and antiangiogenic effects in animals and in humans (13-16). The role of platelets in angiogenesis has now been characterized as mainly supportive of the neovasculature in preventing leakage and hemorrhage (17).

However, the proangiogenic effects of platelets can be counteracted by their capacity to elaborate antiangiogenic factors, including platelet factor 4 (PF4) (18) and thrombospondin 1 (TSP1) (19). Thus, it is unknown whether expression of TSP1 and TSP2 plays a role in determining the angiogenic phenotype of megakaryocytes and platelets.

TSP1 was originally characterized as a "thrombin-sensitive protein with an apparent molecular weight of 190 [kDa]" released from platelet $\alpha$-granules upon activation (20). In the same study, TSP1 was reported to account for $5 \%$ of the platelet fraction's protein content in human peripheral blood preparations and to be exquisitely sensitive to proteolysis. In 1978, the intact homotrimer was isolated from human platelets, and the term thrombospondin was proposed. TSP was not only present in megakaryocytes and platelets but was also found to be a constituent of the extracellular matrix (21). In 1990 TSP1 was the first endogenous inhibitor of angiogenesis to be discovered and characterized (22). Concurrently, a related but distinct protein was identified and named TSP2 (23). At present, the TSP protein family consists of 5 members, 

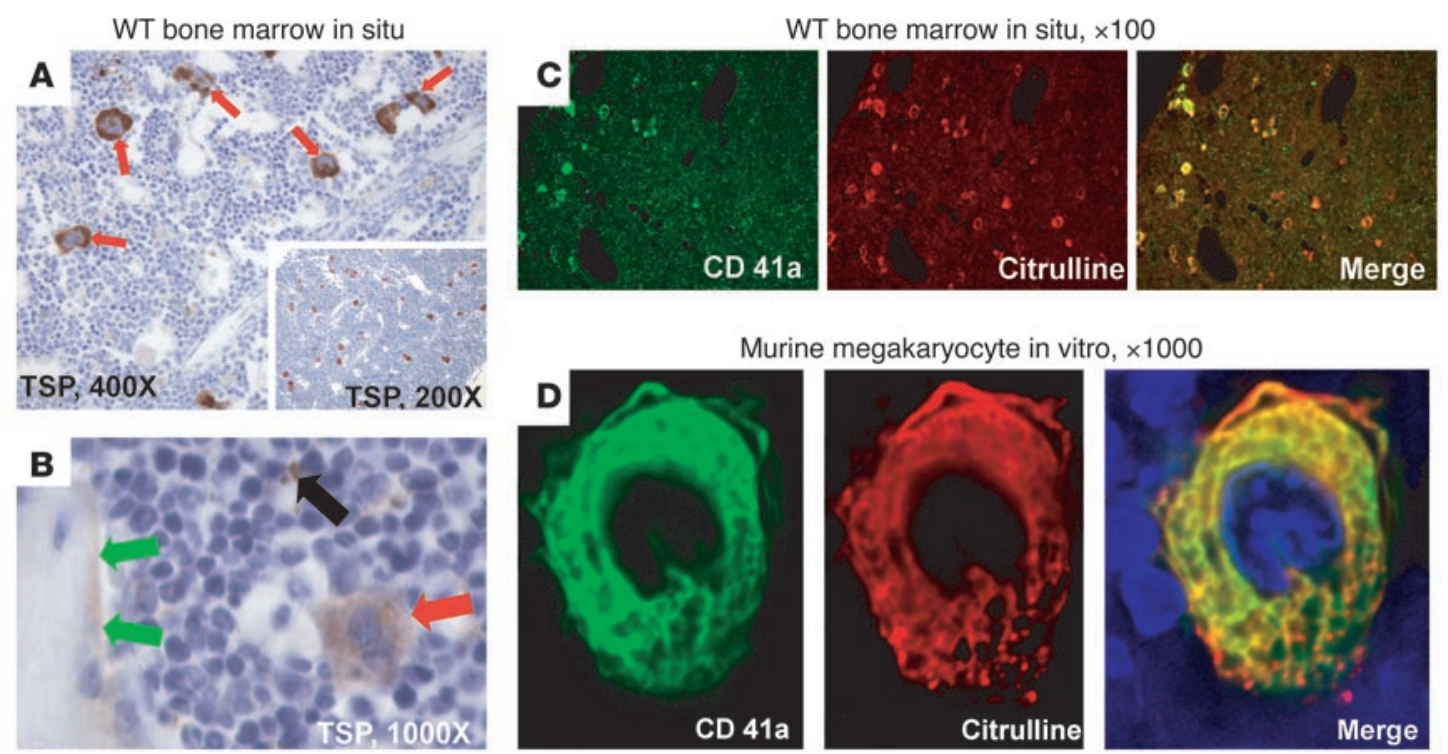

\section{Figure 1}

In the bone marrow, TSP1 is expressed in megakaryocytes and platelets and on endosteal surfaces. An antibody raised against citrullinated proteins results in robust and specific megakaryocyte staining. (A) Megakaryocytes (red arrows) are highly immunoreactive for TSPs. Representative images of WT bone marrow at steady state are shown. Sections were stained for TSPs. Original magnification, $\times 400$. Inset provides a lower magnification overview of WT bone marrow; original magnification, $\times 200$. Sections shown in $\mathbf{A}$ and $\mathbf{B}$ were counterstained with hematoxylin. (B) In addition, TSP immunoreactivity was demonstrated on endosteal osseous surfaces (green arrows) and platelets (black arrow). This would be in line with previous reports in which TSP1 has been shown to be a cytoadhesive molecule for hematopoietic stem cells. On the other hand, hematopoietic cells other than megakaryocytes and platelets did not stain with the same antibody. Frozen bone marrow section at steady state is shown. Original magnification, $\times 1,000$. (C) WT murine steady state bone marrow was stained with an antibody against CD41 and the anti-citrulline antibody. While anti-CD41 binding was visualized with a Cy2-labeled secondary antibody (green), the anti-citrulline antibody was detected with a Cy3-labeled secondary antibody (red). It is obvious that both antibodies bind to megakaryocytes. However, the anti-citrulline antibody results in more robust and stronger staining results. Original magnification, $\times 100$. (D) Murine megakaryocytes differentiated in culture costained with anti-CD41a and anti-citrulline as above. The merged view shows that both antibodies bind to megakaryocytes. Cytospin from in vitro-differentiated lineage negative murine bone marrow cells is shown. Original magnification, $\times 1,000$.

with TSP1 and TSP2 forming homotrimers and TSP3, -4 , and -5 assembling into homopentamers (24). TSPs are classified as matricellular proteins to denote their influence on cellular function and to emphasize that they resemble the extracellular matrix but are not an integral component of extracellular structures (25). TSP1 inhibits migration and proliferation and can induce apoptosis of endothelial cells, possibly mediated through interaction with the endothelial cell receptor CD36 (26). However, its indirect antiangiogenic effects may be more significant than these direct actions (27). Indirect effects include activation of TGF- $\beta$ (28) as well as binding and blockade of activation of MMPs (29). In tumor models, TSP1 is present in high concentrations at the tumor-stroma junction, thereby potentially inhibiting tumor vascularization (30-32).

Platelets contain high quantities of TSP1 and release it upon activation (33), which suggests that release of TSP1 may control the proangiogenic potency of activated platelets. In this study, we describe what we believe is a novel control system by which the angiogenic phenotype of platelets is determined by the absolute number of megakaryocytes and magnitude of TSPs stored within thrombopoietic cells. TSP1 and TSP2 not only negatively regulate megakaryocyte proliferation in the bone marrow and thereby platelet numbers in the peripheral blood, but they also determine bone marrow vascularity as well as the platelet angiogenic phenotype. Our data provide what we believe are novel and important insights into platelet-endothelial cell interactions and their interdependence in the angiogenic process.

\section{Results}

TSP1 expression in bone marrow is restricted to megakaryocytes, platelets, and endosteal surfaces. The precise mechanism whereby localized expression of TSPs may regulate neoangiogenesis is not known. TSPs are not only stored intracellularly but also deposited in the extracellular matrix. To define the mechanism by which TSPs may regulate neoangiogenesis within the marrow, we examined the expression pattern of TSPs within intact marrow sections by immunostaining. TSP1 expression was localized to specific niches within the marrow, including cytoplasm of polyploid megakaryocytes (Figure 1, A and B), platelets (Figure 1B), and endosteal surfaces of both cortical and trabecular bone (Figure 1B). Surprisingly, most of the TSP1 signal came from intracellular stores within these thrombopoietic cells. The majority of TSP $1^{+}$megakaryocytes were found in close apposition to sinusoidal endothelial cells. However, there was little if any detectable TSP1 expression in hematopoietic cells other than megakaryocytes and platelets. Expression of TSP1 proved to be a reliable marker for identification of large polyploid megakaryocytes in both paraffinembedded and frozen bone marrow sections. As control, staining of the marrow of $\mathrm{TSP}^{-/-} \mathrm{TSP}^{-/-}$(TSP-DKO) mice with the same antibody was negative. These data suggest that regional expression of TSPs could modulate marrow vascularity.

A rabbit anti-mouse citrulline antibody serves as a novel specific marker for identification and quantification of polyploid megakaryocytes in the bone marrow. Quantification of polyploid megakaryocytes in WT 


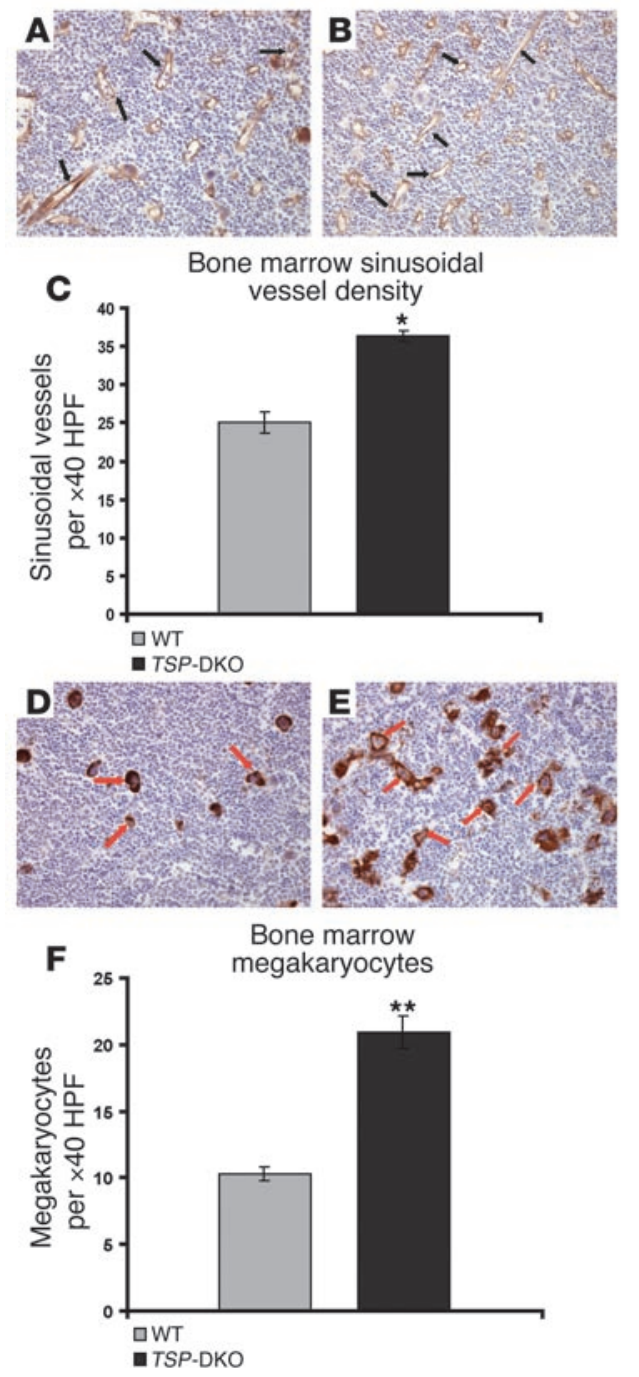

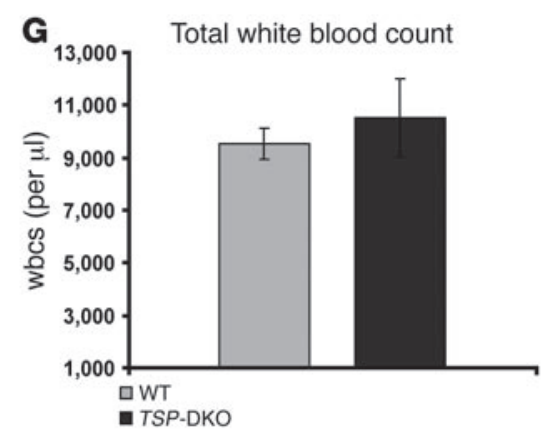
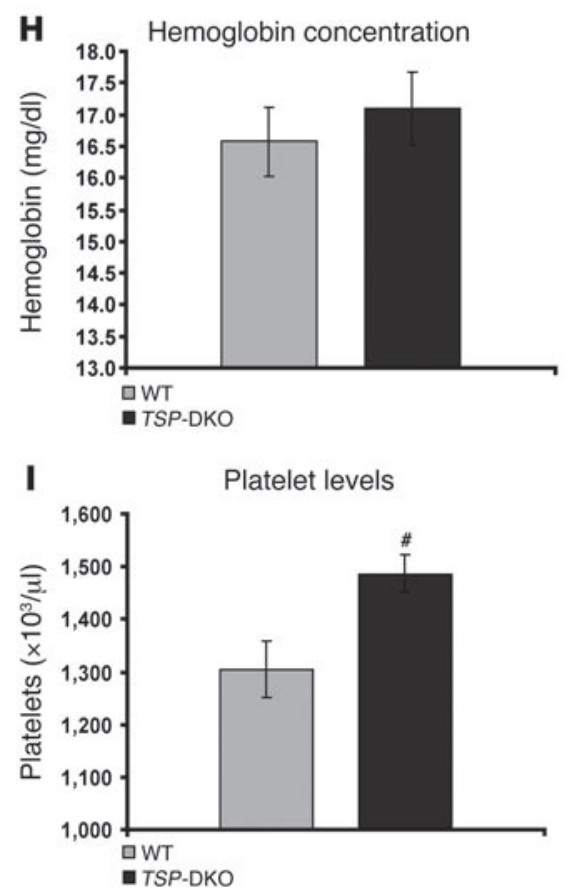

\section{Figure 2}

TSP-DKO mice display increased bone marrow microvascular and megakaryocyte density as well as higher peripheral platelet levels compared with WT mice. (A and B) Marrow sinusoidal microvasculature was quantified in WT (A) and TSP-DKO (B) mice after immunostaining against panendothelial cell antigen clone MECA32. Note that absolute number of cross-sectioned sinusoids (black arrows) is higher in TSP-DKO animals. Representative marrow sections at steady state are shown at original magnification, $\times 400$. DAB was counterstained with hematoxylin. (C) TSP-DKO marrow has a higher sinusoidal microvascular density than WT marrow: $36 \pm 0.7$ versus $25 \pm 1.4$ sinusoidal vessels per field. Scored at original magnification, $\times 400$. HPF, high-power field. ${ }^{*} P<0.005$. (D) WT marrow, stained for TSPs. Note that only megakaryocytes and platelets are stained. Red arrows indicate differentiated, multinucleated megakaryocytes. Original magnification, $\times 400$. DAB was counterstained with hematoxylin. (E) Megakaryocytes in TSP-DKO marrow are abundant at steady state and can be stained with an antibody against citrullinated proteins. This antibody stained megakaryocytes at the same level of differentiation as the TSP antibody (red arrows). Original magnification, $\times 400$. DAB was counterstained with hematoxylin. (F) TSP-DKO megakaryocyte density is almost twice as high as that in WT marrow: $21 \pm 1.2$ versus $0 \pm 0.5$ megakaryocytes per field. Scored at original magnification, $\times 400$. ${ }^{* \star} P<6 \times 10^{-6}$. (G) Leukocyte counts at steady state $(n=6)$. Difference was not significant. (H) Analysis of hemoglobin concentration $(n=6)$ showed similar results. (I) TSP-DKO mice displayed significantly elevated platelet counts compared with WT controls: $1,495,000 \pm 37,000 / \mu$ l versus 1,305,000 $\pm 53,000 / \mu$ l. ${ }^{\#} P<0.05 . n=6$.

mice could readily be accomplished with the use of TSP as the target antigen. However, as subsets of the megakaryocytes may not express TSP, we sought to quantify polyploid megakaryocytes and define their geometrical location in relation to sinusoidal vessels by staining for other antigens. Staining with antibodies against conventional megakaryocyte antigens, including vWF, appeared to either target other structures (e.g., endothelial cells) or reveal that antibodies were not ideally suitable for immunohistochemical staining of intact bone marrow (e.g., CD41; Figure 1C). Therefore, in order to identify polyploid megakaryocytes in TSP-DKO mice, we used what we believe to be a novel, highly specific antibody that targets murine megakaryocytes. This antibody was raised in rabbits against a decapeptide of mouse cytokeratin-1 and binds to highly citrullinated peptides in human cornified skin (34). Staining of sequential sections of WT mouse bone marrow with the anti-TSP1 antibody or anticitrulline antibody showed that the same mature polyploid megakaryocytes from each adjacent section were stained (Figure 1C). Bone marrow sections and cytospins from bone marrow cultures containing hematopoietic cells of all lineages were used to establish the absolute specificity with which the anti-citrulline antibody binds to cells of only the mature polyploidy megakaryocytic lineage (Figure 1D and Supplemental Figure 1; supplemental material available online with this article; doi:10.1172/JCI29314DS1) without cross-reacting with endothelial cells or other nonthrombotic cells. Therefore, the selective expression of a protein recognized by this anticitrulline antibody provides a reliable method for identifying and quantifying TSP-deficient megakaryocytes in cross sections of mouse marrow.

TSP-DKO mice exhibit increased bone marrow microvascular and megakaryocyte density as well as higher platelet levels in the steady state. Examination of the bone marrow revealed a significantly increased microvascular density in TSP-DKO mice (determined by numerical quantification of MECA32 $2^{+}$sinusoids): $36 \pm 0.7$ versus $25 \pm 1.4$ sinusoidal vessels per $\times 400$ high-power field $(P<0.005$; Figure $2, \mathrm{~A}-\mathrm{C})$. MECA32 has previously been found to be equivalent to vascular endothelial cadherin (VE cadherin) as a marker for identifying bone marrow endothelia (35). A major difference was observed when megakaryocytes from TSP-DKO mice were quantified and compared with those of WT mice: the number of polyploid megakaryocytes (Figure 2, D and E) per high-power field was almost 


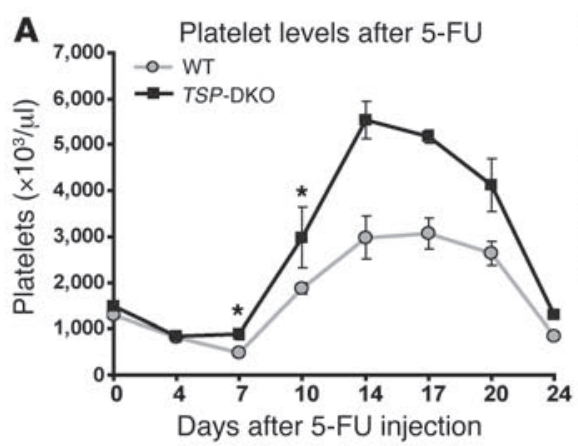

Bone marrow megakaryocytes

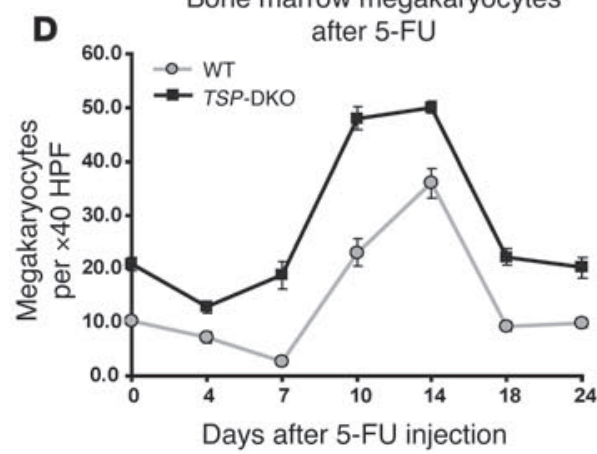

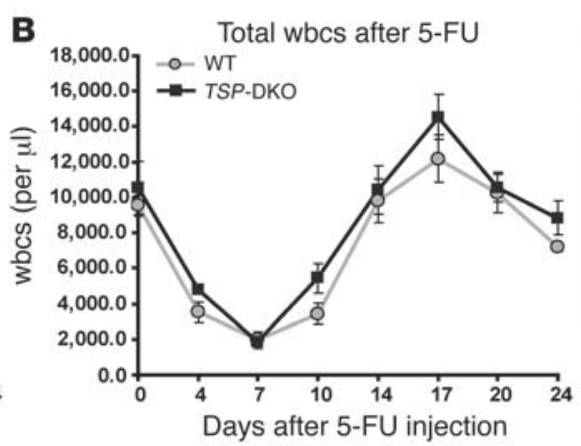

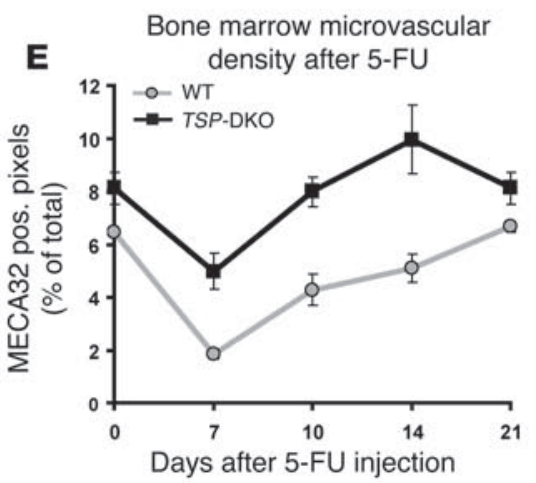

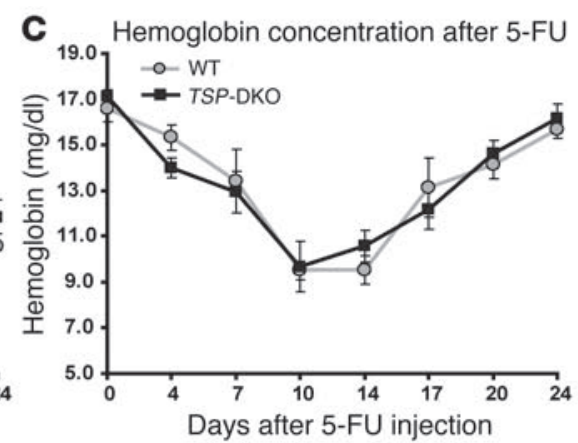

Day 14 after 5-FU

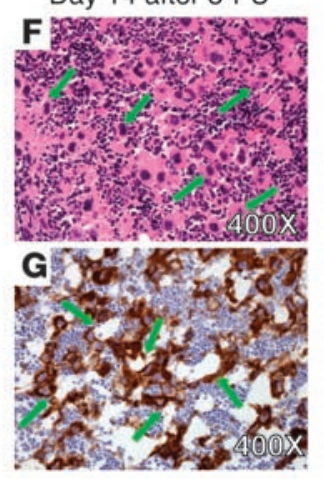

Figure 3

TSP deficiency enhances megakaryocyte repopulation of the bone marrow and platelet production after myelosuppression. (A) After $250 \mathrm{mg} / \mathrm{kg}$ of 5 -FU treatment, WT mice experienced pancytopenia followed by rebound thrombocytosis. Platelets reverted to normal around day 24 . TSP-DKO mice displayed a more rapid regeneration, with $8.9 \times 10^{5}$ platelets/ul compared with $4.77 \times 10^{5}$ platelets/ul in WT animals on day 7 . Furthermore, rebound thrombocytosis was exaggerated, with platelets reaching $5.5 \times 106 / \mu \mathrm{l}$ compared with $2.9 \times 10^{6} / \mu \mathrm{l}$ in WT controls on day $14(n=6$, $P=0.006)$. ${ }^{*} P<0.05$. (B) Leukocytes and $(\mathbf{C})$ hemoglobin concentrations did not show significant differences between TSP-DKO and WT mice $(n=6)$. (D) TSP-DKO mice had higher numbers of megakaryocytes at all time points following 5-FU injection $(P<0.05)$. While megakaryocytes reached their lowest levels on day 7 in WT mice, megakaryocyte concentration returned to normal levels in $T S P$-DKO mice on day $7(P<0.0003)$. (E) After 5-FU injection, endothelial cell mass plummeted to about $2 \%$ of hematopoietic marrow surface area and reverted to normal around day 14. In TSP-DKO mice, the overall course of these changes was similar to that in WT mice. However, MECA32-positive surface area never decreased to less than $4.9 \%$ (day 7 after 5 -FU injection; $P<0.005$ ). ( $\mathbf{F}$ and $\mathbf{G}$ ) The same femoral marrow as represented in $\mathbf{E}$ stained with $\mathrm{H} \& \mathrm{E}$ (F) and anti-citrulline antibody (G). DAB was counterstained with hematoxylin. TSP-DKO marrow showed extreme megakaryocytosis at day 10 after 5-FU injection. Green arrows indicate megakaryocytes. Original magnification, $\times 400$.

twice as high in TSP-DKO animals $(21 \pm 1.2$ versus $10 \pm 0.5$ megakaryocytes per $\times 400$ high-power field, $P<6 \times 10^{-6}$; Figure 2, D-F). Importantly, this latter finding extends in vitro results, indicating that TSPs negatively regulate megakaryopoiesis in bone marrow cultures (36), and underscores previous evidence that bone marrow megakaryocytes and the sinusoidal vasculature are not only spatially but also functionally dependent upon each other (37). Detailed hematological analysis of TSP-DKO mice showed no significant difference in total and differential leukocyte counts (Figure $2 \mathrm{G}$ ). Likewise, erythrocyte count and indices were similar to those of WT control animals (Figure 2H). However, TSP-DKO mice displayed consistently and significantly elevated platelet counts at steady state as compared with WT controls $(1,495,000 \pm 37,000 / \mu \mathrm{l}$ versus $1,305,000 \pm 53,000 / \mu \mathrm{l}, P<0.05$; Figure $2 \mathrm{I})$.

When TSP1 or TSP2 bone marrow sections from single $\mathrm{KO}$ mice were examined, both displayed analogous changes in megakaryocyte or microvascular density, but only the increase in megakaryocytes in TSP1-deficient animals reached significance: $16 \pm 0.6$ megakaryocytes per $\times 400$ high-power field in TSP1-/- mice as compared with $10 \pm 0.5$ in WT mice $\left(P<7 \times 10^{-6}\right)$ and $28 \pm 0.9$ sinusoidal microvessels as compared with $25 \pm 1.4$ in WT mice $(P=0.32)$.
TSP2-deficient mice showed $13 \pm 0.6$ megakaryocytes $(P=0.07)$ and $27 \pm 0.6$ sinusoidal microvessels $(P=0.27)$. This suggests that TSP1 and TSP2 may compensate for each other in the case of animals deficient in a single TSP. Therefore, further experiments were conducted using TSP-DKO mice.

Hemangiogenic regeneration and platelet recovery after myelosuppression are enhanced in TSP-deficient mice. Sex- and age-matched TSP-DKO mice as well as WT control mice were injected i.v. with a myelosuppressive dose of 5 -fluorouracil (5-FU) $(250 \mathrm{mg} / \mathrm{kg})$. Complete blood counts were obtained $4,7,10,14,18,22$, and 28 days afterwards. WT mice displayed a typical course of peripheral pancytopenia followed by a marked rebound thrombocytosis up to approximately $3.0 \times 10^{6} / \mu \mathrm{l}$ that lasted for several days $(n=6)$ before platelet counts reverted to normal levels on approximately day 24 after injection. Similarly, leukocytes reached their nadir around day 7 and reverted back to normal 4 weeks after myelosuppression. However, they did not display a marked rebound leukocytosis (Figure 3, A and B). With TSP-DKO mice, there was a more rapid regeneration of thrombopoiesis with platelet levels of $8.9 \times 10^{5} / \mu \mathrm{l}$ as compared with $4.77 \times 10^{5} / \mu \mathrm{l}$ in WT animals by day $7(P<0.05$; Figure $3 \mathrm{~A})$. Furthermore, rebound thrombocytosis was strongly exaggerated, 

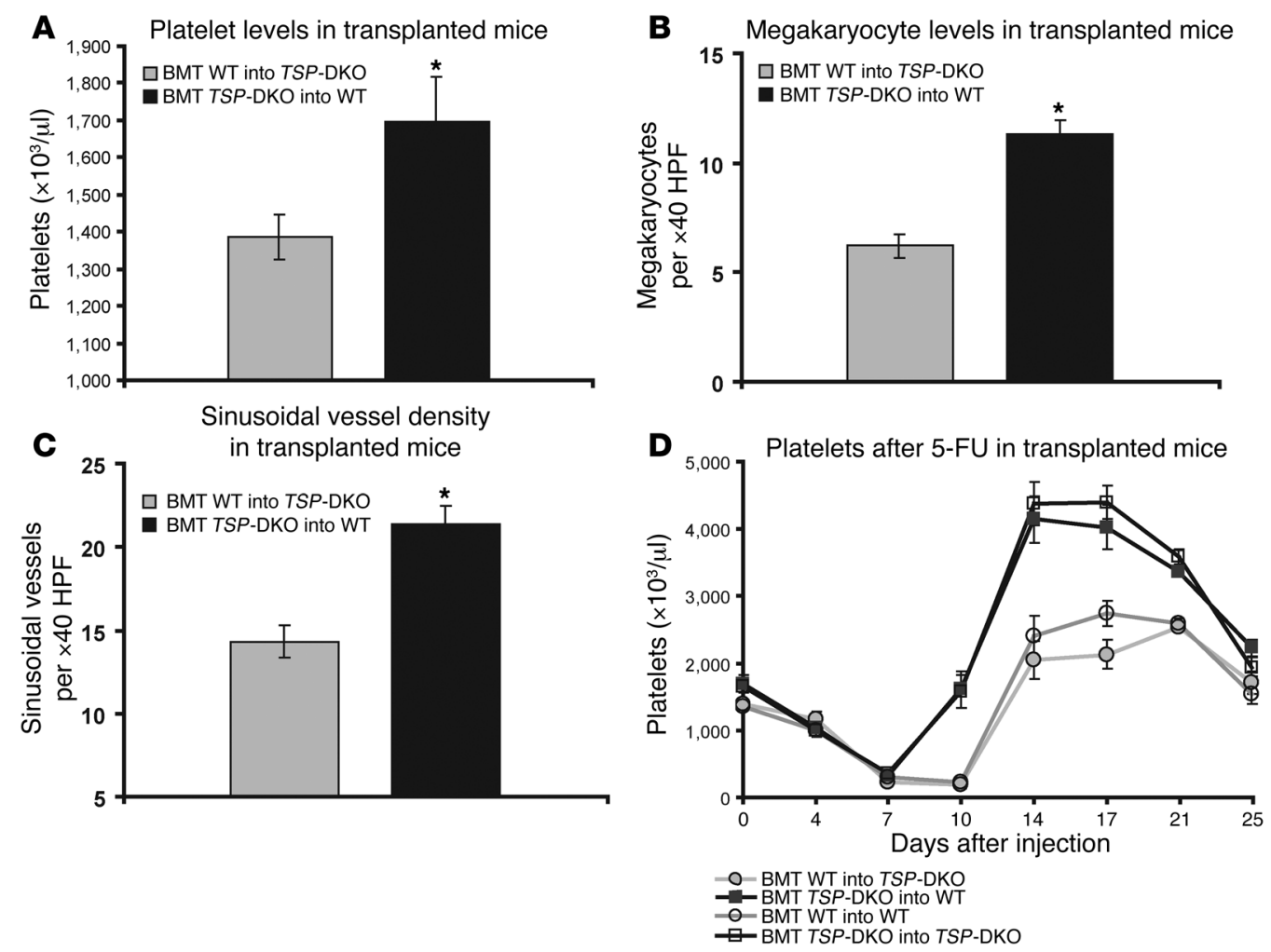

\section{Figure 4}

TSP-deficient thrombocytotic phenotype is transplantable. (A-C) Lethally irradiated (9.5 Gy) WT control mice were transplanted with TSP-DKO bone marrow and TSP-DKO mice were transplanted with WT bone marrow. Ninety days after transplantation, platelet counts were $1.7 \pm 0.1 \times 10^{6} / \mu \mathrm{l}$ in WT recipients transplanted with TSP-DKO bone marrow and $1.4 \pm 0.6106 / \mu \mathrm{l}$ in TSP-DKO recipients transplanted with WT bone marrow. $P<0.04$. Platelet $(\mathbf{A})$ and megakaryocyte levels $(\mathbf{B})$ and vascular density $(\mathbf{C})$ were determined in the transplanted mice. (D) When 5-FU was given (250 mg/kg i.v.) to the same mice, WT mice with TSP-deficient hematopoiesis displayed a faster platelet recovery than TSP-deficient mice that had been transplanted with WT bone marrow. On day 10, platelet levels were $0.18 \pm 0.05 \times 106 / \mu l$ in TSP-DKO mice with WT hematopoiesis compared with $1.6 \pm 0.27 \times 106 / \mu$ in the WT recipients of TSP-deficient bone marrow $(P<0.03)$. Therefore, TSP from transplanted megakaryocytes, rather than TSP presented or secreted by nontransplanted bone marrow stromal components, may regulate megakaryopoiesis and thrombopoiesis following myelosuppression. ${ }^{*} P<0.05$.

with platelet levels reaching an average of $5.5 \times 10^{6} / \mu \mathrm{l}$ as compared with $2.9 \times 10^{6} / \mu \mathrm{l}$ in WT controls on day 14 following 5 -FU injection $(n=6, P=0.006$; Figure $3 \mathrm{~A})$. The phenomenon of thrombocytosis after 5 -FU injection is well described $(35,38)$ and reflects an increased number of megakaryocytes in the bone marrow. This suggests that an increased number of progenitors differentiate into megakaryocytes (39). Splenic megakaryopoiesis reportedly is not a major contributor to rebound thrombocytosis after 5-FU injection (40). There were no significant differences when total leukocyte counts and hemoglobin concentrations were compared between TSP-DKO and WT control mice ( $n=6$; Figure 3 , B and C).

Histological analyses of bone marrow demonstrated higher numbers of megakaryocytes at any given time point in TSP-DKO mice. Indeed, at day 7 following myelosuppression, when the WT megakaryocyte population reached its nadir, TSP-DKO bone marrow already displayed an upward trend in megakaryocyte number (Figure 3D). In addition, microvascular density was measured by the number of pixels showing positive staining for the panendothelial marker MECA32 on digitally acquired images of the hematopoietic marrow. As we have demonstrated, myelosuppressive agents not only target cycling hematopoietic cells but also lead to loss of microvascular integrity and of sinusoidal endothelial cell mass in the marrow (35). Indeed, at the time of the nadir, there was no single unaffected vascular sinusoid to be found in the marrow. In both, WT and TSP-DKO mice, the overall time course of these changes correlated with the number of megakaryocytes in the bone marrow (Figure 3, D and E). When TSP-deficient bone marrow was analyzed 14 days after 5-FU injection, the megakaryocyte content was strikingly high (Figure $3 \mathrm{~F}$ ). These results indicate that TSP is an endogenous inhibitor of excessive megakaryocyte proliferation in vivo and consequently regulates circulating platelet levels. In addition, bone marrow's sinusoidal microvascular density correlated with its megakaryocyte content.

TSP expressed by hematopoietic cells, rather than that expressed by stromal cells, confers the angiogenic and thrombocytotic phenotype. We sought to establish the role of megakaryocytes versus the bone marrow stromal compartment in the enhanced thrombocytopoiesis phenotype following myelosuppression. Thus, lethally irradiated WT control mice were transplanted with TSP-DKO bone marrow, and TSP-DKO mice were transplanted with WT bone marrow. Ninety days after transplantation, WT recipients showed higher platelet counts than TSP-DKO recipients $(1,695,100 \pm 122,000 / \mu \mathrm{l}$ in WT recipients transplanted with TSP-DKO bone marrow versus $1,385,500 \pm 59,000 / \mu \mathrm{l}$ in TSP-DKO recipients transplanted 
A
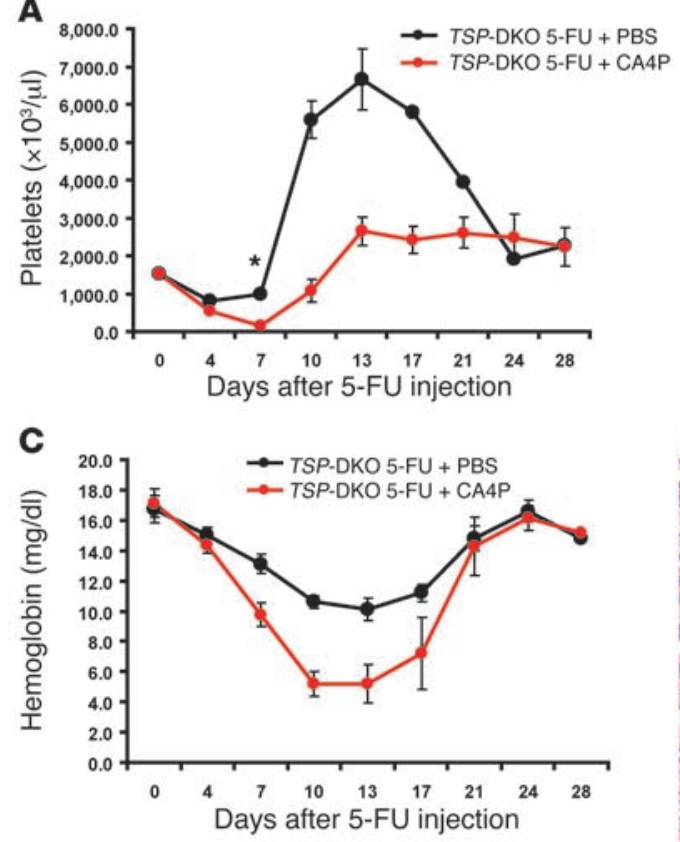
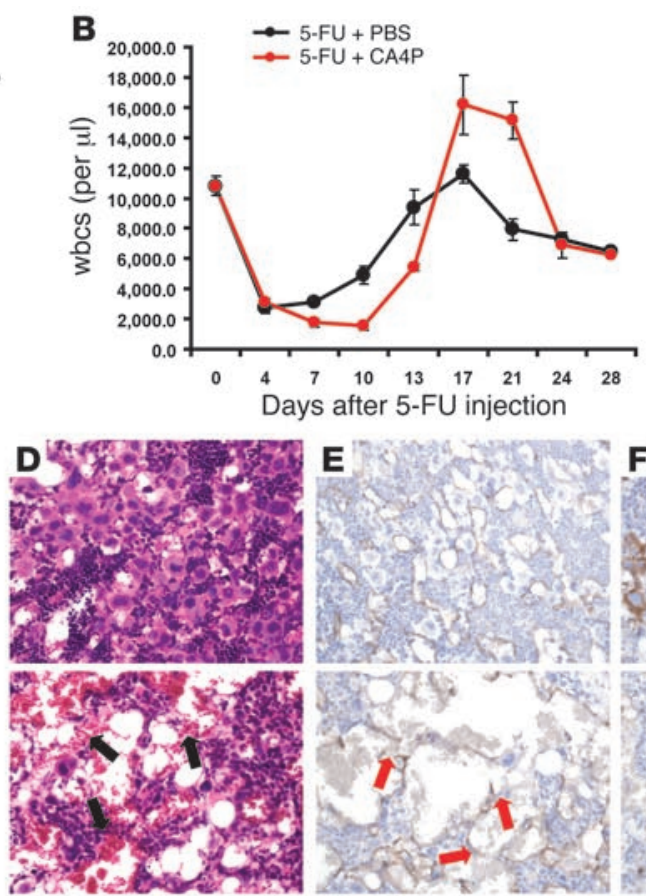

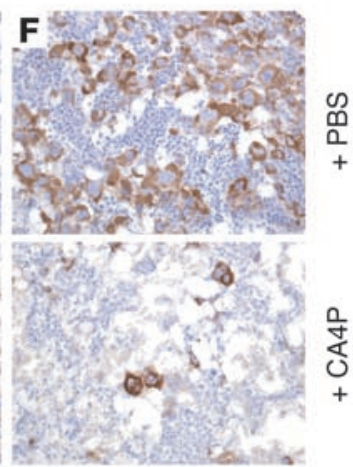

\section{Figure 5}

The vascular disrupting agent CA4P targets bone marrow microvascular sinusoids as well as megakaryopoiesis. (A) CA4P delays platelet recovery and blocks rebound thrombocytosis. TSP-DKO mice were treated with $250 \mathrm{mg} / \mathrm{kg} 5$-FU i.v. and either with an additional low dose of CA4P $(25 \mathrm{mg} / \mathrm{kg}$ ) every other day or with PBS. CA4P has not been shown to be myelosuppressive at this dose in long-term studies (data not shown). While 5-FU alone resulted in a typical rebound thrombocytosis, CA4P-treated mice reverted back to normal platelet levels later, and the rebound effect was completely abrogated. $n=5$ in each group. $P<0.02$ on day 13 . (B) CA4P delays white blood cell recovery. Total peripheral blood white cell counts displayed a lower nadir and a delayed recovery albeit with a marked rebound effect that is rather atypical for white cells. (C) CA4P increases severity of anemia but does not delay red blood cell recovery. Hemoglobin levels were decreased to lower absolute levels when CA4P was added to the myelosuppressive regimen, but the overall time course of rbc regeneration was comparable to that in the group that received 5-FU only. (D) Histological analysis of the marrow on day 10 revealed that the repopulation of the marrow with hematopoietic cells was not significantly inhibited. However, the vascular microarchitecture was severely disturbed, resulting in areas of hemorrhage (black arrows). H\&E staining was used. Original magnification, $\times 400$. (E) Staining with the panendothelial cell marker antibody MECA32 showed disrupted, leaky microvasculature in the CA4P-treated mice (red arrows). DAB was counterstained with hematoxylin. Original magnification, $\times 400$. (F) The same femurs as in $\mathbf{D}$ were stained with anti-citrulline antibody to identify megakaryocytes. Interestingly, it became evident that the vascular disruption seen with CA4P treatment resulted in a selective defect in megakaryocyte repopulation of the marrow, with a reversion of the TSP-DKO phenotype toward normal. Anti-citrulline staining with DAB was counterstained with hematoxylin. Original magnification, $\times 400$.

with WT bone marrow; $P<0.04$; Figure 4A). Analysis of the femoral bone marrow revealed that there were significantly higher concentrations of megakaryocytes when hematopoietic marrow of WT recipients of TSP-DKO marrow was compared with that of TSP-DKO recipients of WT marrow $(11 \pm 0.6$ versus $6 \pm 0.5$ megakaryocytes per $\times 400$ high-power field image of hematopoietic marrow; $P<0.0004$; Figure 4B); this was similar to our findings in the steady state. Increased megakaryocytes correlated with increased microvascular density as measured by quantification of sinusoids ( $21 \pm 1.0$ versus $14 \pm 1$ sinusoidal vessels per $\times 400$ highpower field of hematopoietic marrow; $P<0.0001$; Figure 4C); this is similar to our observations in the steady state. The fact that the absolute numbers in these transplanted mice are lower compared with those in the mice examined under steady state conditions reflects the lower overall cellularity of the marrow after lethal irradiation and transplantation.

In addition, when treated with 5 -FU $(250 \mathrm{mg} / \mathrm{kg}$ i.v. $)$, WT mice with TSP-DKO hematopoiesis displayed a faster platelet recovery than TSP-DKO mice transplanted with WT bone marrow. On day 10 after 5-FU injection, TSP-DKO recipients had a platelet count of $184,800 \pm 46.000 / \mu \mathrm{l}$ as compared with $1,608,000 \pm 270.000 / \mu \mathrm{l}$ in WT recipients of TSP-deficient bone marrow $(n=6, P<0.03$; Figure 4D). Note that the outcome in control transplantations closely matches the corresponding transplantations of transgenic marrow into WT marrow and vice versa, confirming that transplantable cells capable of releasing TSPs regulate peripheral platelet levels after 5-FU treatment. These results establish that megakaryocytederived TSPs are responsible for repression of megakaryopoiesis and thrombopoiesis after myelosuppression. This is in preference to TSPs presented or secreted by nontransplanted bone marrow stromal components. Therefore, the functional significance of the cellular sources of TSPs from thrombopoietic cells is consistent with our histological findings, which suggest that in the steady state, the majority of TSP is confined to intracellular stores.

The vascular disrupting agent combretastatin A4 phosphate targets regenerating bone marrow vascular endothelium and abolishes rebound megakaryocytosis. Bone marrow microvascular endothelium is morphologically and functionally unique in that it provides both a conduit for mobilization of hematopoietic cells to the peripheral circulation and a catalytic surface for the differentiation of 
A

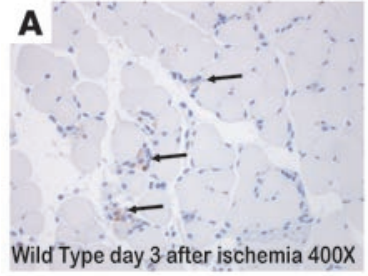

B

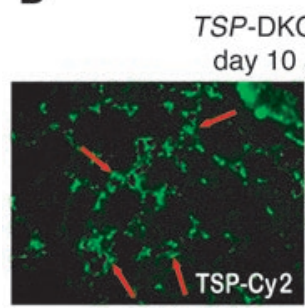

TSP deposition:

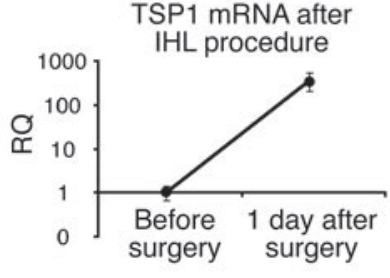

SP1 mRNA after

WT platelets,
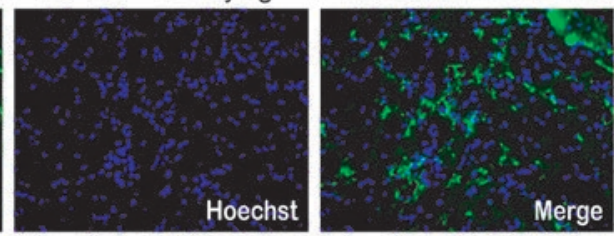

Nonischemic musculature of the same animal $\times 200$
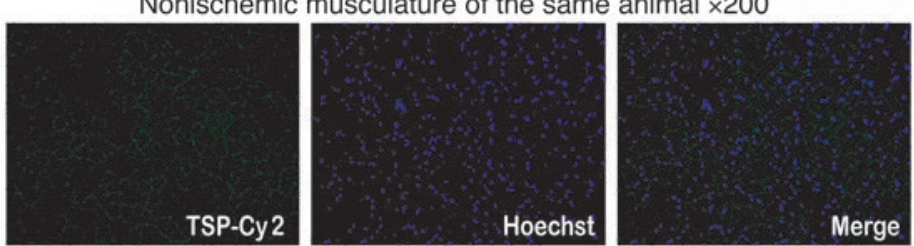

Figure 6

TSPs are deposited perivascularly in ischemic musculature. (A) Left: WT mice underwent ligation of the left femoral artery. Shortly after the surgical procedure, ischemic tissue of the affected limb's gastrocnemius muscle was harvested. Immunohistochemical analysis of the ischemic areas showed deposition of TSP in and around microvessels (black arrows). Note the perivascular location of freshly deposited TSPs along with inflammatory cells infiltrating the ischemic musculature. Importantly, there was no detectable staining on nonischemic musculature. Paraffin-embedded section from a representative field of WT gastrocnemius after femoral vessel ligation, stained with $\mathrm{DAB}$ and counterstained with hematoxylin. Scored at original magnification, $\times 400$. IHL, ischemic hind limb. Right: RNA was extracted from ischemic gastrocnemius tissue, and TSP1 mRNA levels were compared with those at steady state. A strong increase in relative expression of TSP1 was found at the mRNA level $\left(2^{-\Delta \mathrm{Ct}} \times 10,000\right)$. This finding is in line with previous observations in skin healing models, where TSP1 mRNA is thought to be derived from invading hematopoietic cells. $\mathrm{RQ}$, relative expression. (B) TSP-DKO mice 3 days after ischemic hind limb surgery received a single transfusion with $300 \times 10^{6}$ WT platelets. Seven days later, TSPs were deposited perivascularly only in ischemic musculature (red arrows). Frozen section, representative field of ischemic TSP-DKO gastrocnemius muscle, stained for TSPs with Cy2-conjugated secondary antibody and Hoechst 33342 nuclear stain. Scored at original magnification, $\times 400$. As an internal control for autofluorescence and background staining, nonischemic musculature of the contralateral, nonischemic gastrocnemius muscle stained for TSP is shown. Original magnification, $\times 200$.

hematopoietic progenitor cells in the bone marrow $(37,41)$. In turn, hematopoietic cells, including megakaryocytes, support the discontinuous sinusoidal bone marrow endothelium by releasing pro- or antiangiogenic factors $(5,42)$. We therefore hypothesized that the increased microvascular density in TSP-DKO mice is due to the transition of megakaryocytes into a proangiogenic phenotype resulting from the lack of TSPs. After a myelosuppressive dose of radiation or 5-FU, the bone marrow's vascular network regresses to a point where normal sinusoidal vessels can no longer be detected (43). Indeed, the recovery of vascular structures in the marrow, i.e., angiogenesis, is a prerequisite for the regeneration of hematopoiesis (35). Therefore, hemangiogenic regeneration after myelosuppression can be studied as an assay of bone marrow angiogenesis. We hypothesized that inhibition of vascular recovery in the bone marrow after myelosuppression should result in impaired megakaryocytic and thrombocytic regeneration. To verify this, TSP-DKO mice were injected i.v. with $250 \mathrm{mg} / \mathrm{kg} 5$-FU. Then the animals received either a nonmyelosuppressive dose of combretastatin A4 phosphate (CA4P) $(25 \mathrm{mg} / \mathrm{kg})$ or PBS as control every other day. Control mice that were injected with the same dose of CA4P alone over the course of the experiment did not develop cytopenias of any kind $(n=4)$. This finding is in line with previous work from our group, in which CA4P has been found to selectively target the assembly of nonstabilized neovessels by rapid functional inhibition of the VE cadherin/ $\beta$-catenin complex as needed for endothelial cellcell adhesion and survival during neovessel assembly and remodeling (44). An effect on hematopoiesis at a low dose of $25 \mathrm{mg} / \mathrm{kg}$ was therefore not to be expected. However, hemangiogenic recovery, because it is dependent on angiogenesis, was anticipated to be impaired. Indeed, the nadir of all blood cell lineages was lower in the group that was treated with CA4P in addition to 5-FU (Figure 5, A-C). Nevertheless, marrow regenerated in all of the treated mice. Remarkably, rebound thrombocytosis was completely blunted in the CA4P-treated mice. Histological analyses of the bone marrow demonstrated hemorrhagic changes (Figure 5D) and disrupted vasculature at day 10 following 5-FU treatment (Figure 5E). However, the difference in megakaryocyte content was most striking. While megakaryocytes were abundant in the mice treated only with 5-FU, there was a selective depletion of bone marrow megakaryocytes in the CA4P-treated group (Figure 5F). The latter difference was still apparent at day 14 after 5-FU treatment, at which time the bone marrow appeared normocellular in both treatment groups (data not shown). However, the CA4P-treated group showed a paucity of megakaryocytes, and immunohistochemical analyses of the sinusoidal vasculature revealed vessel disorganization and lower microvascular density. These data support the hypothesis of a mutual interdependence between the bone marrow's sinusoidal microvasculature and megakaryocytes. The dependence of megakaryocytes on functioning sinusoidal endothelium was so crucial that targeted disruption of vascular remodeling following myelosuppression resulted in selective loss of megakaryocytes and inhibited the TSP-deficient thrombocytotic phenotype. In addition, platelet levels in these mice followed a time course similar to that of WT mice. When 2 groups of TSP-producing WT mice $(n=5$, each) were treated using the same regimens (5-FU with and without CA4P), rebound thrombocytosis was reduced to subnormal levels, and hematopoietic regeneration was delayed in the CA4P-treated group (data not shown).

Platelet-derived TSPs accumulate in areas of ischemia. Since megakaryocyte-derived TSP1 impedes bone marrow microvascular recovery and diminishes regeneration of thrombopoiesis, platelet-derived TSPs might affect peripheral angiogenic processes as well. To test this hypothesis, we examined the rate and extent of revascularization after femoral artery ligation in WT and TSP-DKO mice (45). Analyses of TSP expression in ischemic as compared with nonischemic lower limb muscles of the WT mice 
WT
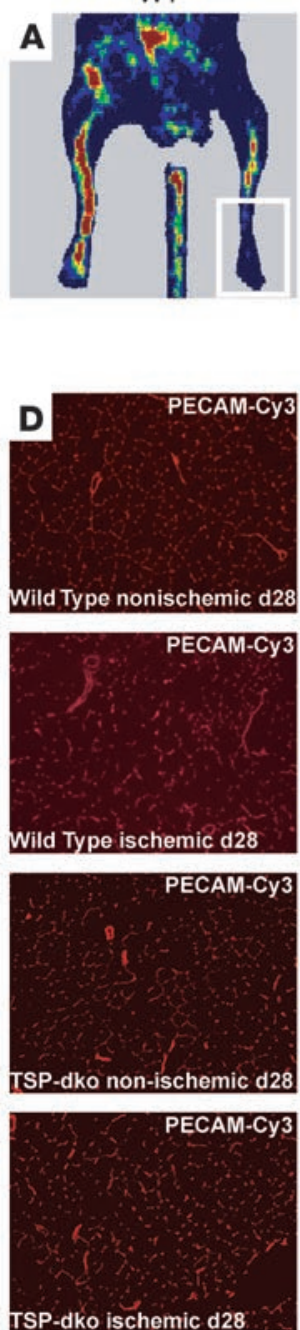

TSP-DKO
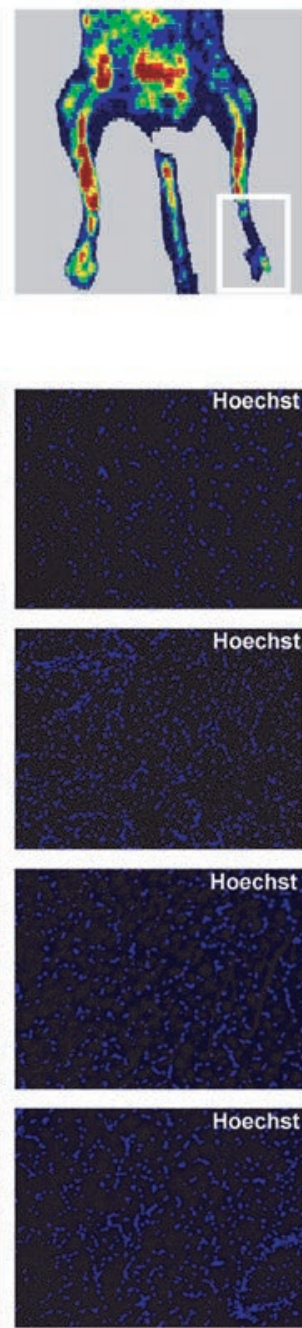

B

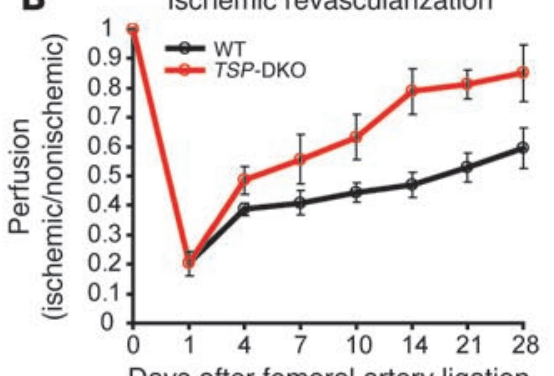

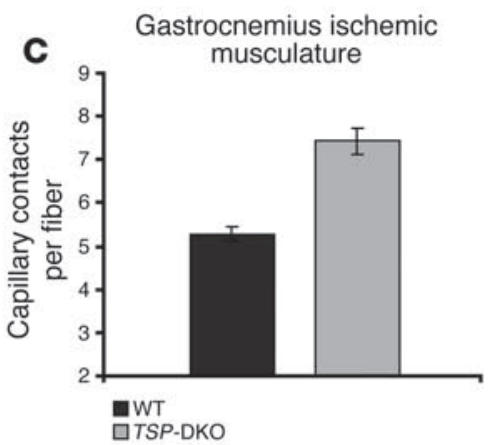
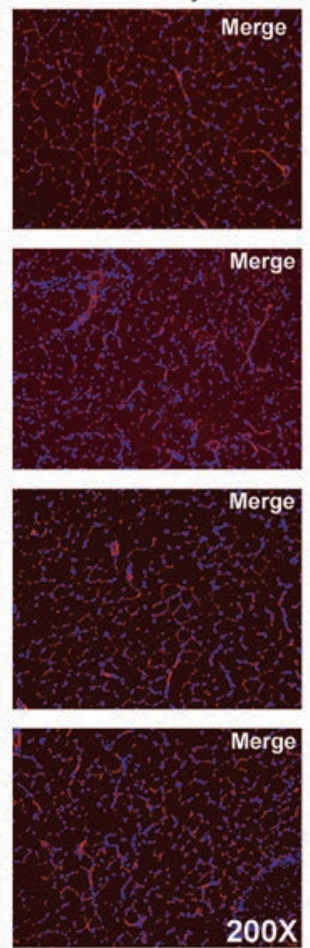

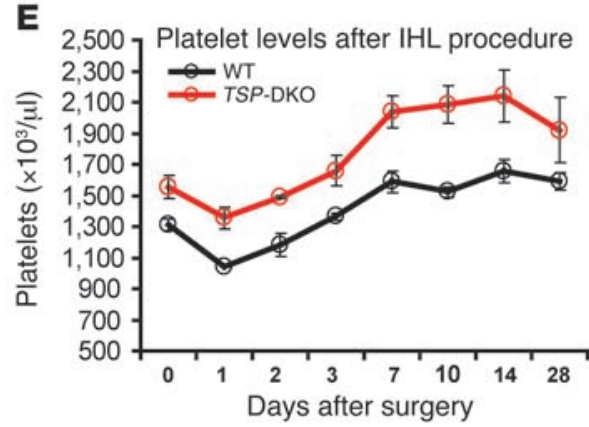

Ischemic revascularization

$\mathbf{F}$ after transplantation

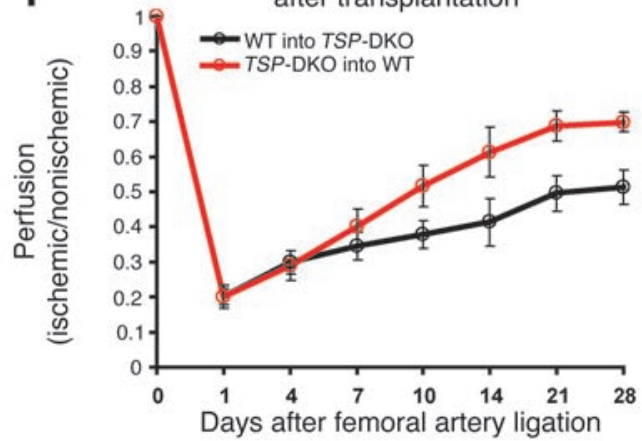

Figure 7

TSP deficiency results in enhanced recovery and neoangiogenesis after induction of hind limb ischemia. (A) Representative color-coded Doppler flow diagram at day 28 following ligation of the left femoral artery in a WT and a TSP-DKO mouse. Differences in perfusion are rendered visible. The selected area of interest is outlined in white. (B) When recovery of perfusion is measured as a ratio of blood flow on the ligated and the nonligated sides, the difference between WT and TSP-DKO mice becomes evident. Beginning at day 4 after surgery, TSP-DKO animals showed increased perfusion rates $(n=6, P<0.05$ at day 4$)$. (C) Microvascular density was higher in ischemic gastrocnemius 28 days after hind limb ischemia in TSP-DKO mice $(n=4, P<0.05)$. (D) Microvascular density as measured by PECAM-1 (CD31) immunofluorescent staining in ischemic gastrocnemius indicates that TSP-DKO mice display increased muscular microvascular density. Microvascular density in the nonischemic gastrocnemius is shown for comparison. Original magnification $\times 200$. (E) TSP-DKO mice develop higher platelet levels following hind limb ischemia ( $n=6, P<0.05$ on all time points). (F) The TSP-deficient, proangiogenic phenotype is transplantable. WT mice were transplanted with TSP-DKO marrow, and TSP-DKO mice were transplanted with WT marrow. Six weeks after transplantation, all mice underwent ischemic hind limb surgery. Reperfusion was slower after lethal irradiation and transplantation compared with that in mice operated on in the steady state. However, when the groups of transplanted animals were compared, it became evident that TSP-DKO marrow conferred the proangiogenic phenotype to the recipient mouse.

demonstrated increased TSP expression in ischemic tissue at the 24-hour time point, at both the RNA (Supplemental Figure 2) and protein levels (Figure 6A). Similarly, in wound-healing models, an early increase in TSP1 was observed during the inflammatory phase of the regeneration process (46). This raises the question of whether this increase was due to locally increased transcription of TSP1 in ischemic tissue or whether the TSP1 was platelet derived. Platelets, although anucleate, contain substan- tial quantities of mRNAs, derived from megakaryocytes $(47,48)$. These mRNAs contribute substantially to RNA preparations obtained from tissue homogenates. In addition, although platelets receive most of their proteome from megakaryocytes, they exhibit limited protein synthesis (49) and, moreover, release substantial amounts of TSP1 protein upon activation (33). In order to clarify whether TSP was delivered by platelets rather than produced by the ischemic tissue, TSP-DKO mice underwent ischemic 


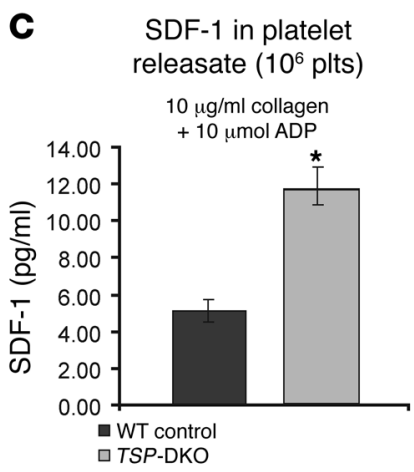

\section{Figure 8}

TSP-deficient platelets release higher amounts of SDF-1 after stimulation. (A) SDF-1 levels in carefully collected platelet-poor plasma were measured. However, most samples contained SDF-1 below detection level. An average concentration of less than $250 \mathrm{pg} / \mathrm{ml}$ was calculated for both WT and TSP-DKO animals $(n=6)$. (B) Retro-orbital blood from the same animals as in A was collected and incubated for clot formation, and serum was harvested after centrifugation. SDF-1 in the serum was higher in TSP-DKO than in WT blood. However, this difference did not reach a level of significance $(2.3 \mathrm{ng} / \mathrm{ml}$ in WT versus $2.8 \mathrm{ng} / \mathrm{ml}$ in TSP-DKO serum; $P=0.08)$ but reflected the elevated platelet levels found in TSP-DKO mice. Importantly, these results strongly suggest that platelets are the major source of serum SDF-1. (C) PRP underwent analysis by aggregometry, and SDF-1 released upon stimulation with different platelet agonists was examined. Stimulation of $10 \mu \mathrm{g} / \mathrm{ml}$ collagen followed by $10 \mu \mathrm{mol}$ adenosine resulted in the strongest aggregation. Interestingly, TSP-DKO platelets secreted twice as much total SDF-1 as WT platelets under these conditions $\left(5 \mathrm{pg} / \mathrm{ml}\right.$ in WT versus $11.5 \mathrm{pg} / \mathrm{ml}$ in TSP-DKO). PRP was pooled from $n=3-4$ animals, experiment was repeated 3 times, $\left.{ }^{\star} P<0.05\right)$.

hind limb surgery and received a single transfusion with $300 \times 10^{6}$ WT platelets on day 3 after surgery. Seven days later, the mice were anesthetized and perfused, and ischemic musculature was harvested and stained for the presence of TSPs. If TSPs were present, their origin would have to have been the transfused, TSPcontaining WT platelets. Indeed, whereas control tissue from the nonischemic legs of these TSP-DKO mice did not stain for TSPs, the ischemic lower limb musculature displayed deposition of TSPs around microvessels (Figure 6B). Green background fluorescence has been found to be problematical in the histological examination of skeletal muscle (50). However, control tissue derived from the contralateral, nonischemic leg of the same TSP-DKO animal after transfusion of WT platelets, showed no significant increase in background fluorescence.

The deployment of TSPs from platelets in ischemic, remodeling microvascular beds has not, to our knowledge, been demonstrated before, and its presence may modulate neoangiogenic processes in response to ischemia and microvascular injury. In order to provide further evidence that deposited platelets are indeed the source of TSPs, we examined the ischemic musculature of WT mice for the expression of platelet-specific marker CD41 (also known as GPIIb). Staining revealed colocalization of CD41 and TSP in ischemic neovessels (Supplemental Figure 4). This shows that platelet constituents themselves are deposited in addition to their contents along ischemic microvascular beds.

Revascularization of ischemic limbs is accelerated in TSP-DKO mice. To formally assess the impact of TSP1 and TSP2 deficiency on the regulation of revascularization, the femoral arteries of TSP-DKO and WT mice were ligated, and the extent of revascularization was determined. To this end, we used color-coded Doppler examination with an area of interest corresponding to the foot region of both the ischemic and the nonischemic legs at specified time points after surgery (Figure 7A). As expected, revascularization of the ischemic limbs was significantly faster in TSP-DKO than in WT mice (Figure 7B). Histological analysis showed increased microvascular density in the musculature of the distal ischemic limb in TSP-DKO mice (Figure 7, C and D). In addition, TSP-DKO mice displayed persistently elevated platelet counts as compared with WT controls throughout the experiment (Figure 7E). However, the overall pattern of changes in platelet counts was similar to that of controls and was characterized by an initial thrombocytopenic phase followed by a slight increase and finally reversion back to steady state levels about 3 weeks after femoral artery dissection (Figure 7E). These findings confirm that there are quantitative abnormalities in terms of enhanced thrombopoiesis in TSP-DKO animals and are in line with previous observations that quantitative increases in platelets support faster recovery in the model of hind limb ischemia (8).

Moreover, when transplanted animals were used for the same experiment, we found the proangiogenic TSP-DKO phenotype to be transplantable. Whereas overall ischemic recovery was delayed in comparison with that in animals that had undergone ischemic hind limb surgery in the steady state, there was still significantly enhanced reperfusion of WT animals that had been transfused with TSP-DKO marrow as compared with reperfusion of TSP-DKO animals that had been transfused with WT marrow (Figure 7F). These data indicate that TSP 1 and TSP 2 released by the platelets may exert a paracrine antiangiogenic effect or a modulating action on platelet function through an autocrine mechanism.

TSP determines the platelet angiogenic phenotype by altering the absolute and differential release of stromal-derived factor 1. Platelets are known to bind to neoangiogenic vascular walls (17) and support angiogenesis by releasing proangiogenic factors. We have shown that the proangiogenic effect of VEGF-A is mediated in part through deployment of stromal cell-derived factor 1 (SDF-1) (CXCL12) released from platelets (45). In addition, SDF-1 has been shown to induce blood vessel formation when administered subcutaneously. SDF-1 enhances recruitment of endothelial progenitor cells as well as proangiogenic hematopoietic cells into ischemic tissue (51-53). To determine whether TSPs could modulate SDF-1 release, the capacity of platelets isolated from TSP-DKO mice to release SDF-1 was determined and compared with that in WT mice. When SDF-1 


\section{Table 1}

TSP-DKO platelets differentially release higher quantities of SDF-1

\begin{tabular}{|c|c|c|c|c|c|}
\hline \multirow[b]{2}{*}{ Stimulants } & $5 H T$ released $(\%)$ & \multicolumn{2}{|c|}{ SDF-1 released (\%) } & \multicolumn{2}{|c|}{ Ratio SDF-1/5HT } \\
\hline & TSP-DKO & WT & TSP-DKO & WT & TSP-DKO \\
\hline Collagen $(5 \mu \mathrm{g} / \mathrm{ml})$ & $46.3 \pm 3.7 \quad 34.3 \pm 5.5$ & $23.5 \pm 2.1$ & $28 \pm 0.7$ & $0.51 \pm 0.07$ & $0.89 \pm 0.11$ \\
\hline$P$ values & 0.26 & \multicolumn{2}{|c|}{0.30} & \multicolumn{2}{|c|}{$<0.05$} \\
\hline Collagen $(10 \mu \mathrm{g} / \mathrm{ml})$ & $50 \pm 1.4 \quad 45.6 \pm 1.9$ & $29.4 \pm 2.8$ & $36.3 \pm 2.7$ & $0.59 \pm 0.06$ & $0.8 \pm 0.06$ \\
\hline$P$ values & 0.23 & \multicolumn{2}{|c|}{0.23} & \multicolumn{2}{|c|}{$<0.05$} \\
\hline Collagen $(5 \mu \mathrm{g} / \mathrm{ml})+\mathrm{ADP}(5 \mu \mathrm{mol})$ & $52.7 \pm 1.4 \quad 49.2 \pm 2.8$ & $30.4 \pm 2.5$ & $35.6 \pm 2.4$ & $0.58 \pm 0.06$ & $0.75 \pm 0.08$ \\
\hline$P$ values & 0.48 & \multicolumn{2}{|c|}{0.28} & \multicolumn{2}{|c|}{$<0.05$} \\
\hline Collagen $(10 \mu \mathrm{g} / \mathrm{ml})+\operatorname{ADP}(10 \mu \mathrm{mol})$ & $56.25 \pm 2.9 \quad 55.2 \pm 2.5$ & $31.5 \pm 3.2$ & $39.9 \pm 2.6$ & $0.54 \pm 0.03$ & $0.73 \pm 0.05$ \\
\hline$P$ values & 0.91 & \multicolumn{2}{|c|}{0.15} & \multicolumn{2}{|c|}{$<0.05$} \\
\hline
\end{tabular}

Platelet aggregometry with WT versus TSP-DKO platelets indicated that the intensity of stimulation directly correlated with the percentage of serotonin or SDF-1 released from the platelets. High-dose collagen followed by high-dose ADP provided the strongest stimulus and resulted in the release of $56.25 \%$ (WT) versus $55.2 \%$ (TSP-DKO) of their ${ }^{14} \mathrm{C}$-serotonin and of $31.5 \%$ (WT) versus $39.9 \%$ (TSP-DKO) of their SDF-1 content. Shown are the means of 4 independent experiments with PRP pooled from $n=3-4$ mice per genotype for each experiment. Interestingly, TSP-DKO platelets differentially secreted not only higher quantities of SDF-1 but also a higher percentage of their total SDF-1 content as normalized to the percentage of serotonin released upon stimulation (SDF-1/serotonin ratio of $0.72-0.82$ in TSP-DKO versus $0.51-0.59$ in WT, $P<0.05$ in each group).

levels were measured in carefully prepared platelet-poor plasma of WT and TSP-DKO mice, no significant differences were observed. In fact, in these experiments, SDF-1 levels in plasma were undetectable in most samples (Figure 8A; $n=6$ ). In contrast, when serum levels were compared in WT and TSP-DKO animals, the latter displayed slightly elevated SDF-1 levels $(2.31 \pm 0.08 \mathrm{ng} / \mathrm{ml}$ versus $2.83 \pm 0.12 \mathrm{ng} / \mathrm{ml} ; P=0.08$; Figure $8 \mathrm{~B})$. The difference did not reach a level of significance and reflected the higher platelet numbers in TSP-DKO mice as described above. However, these data indicate that platelets are not only able to secrete SDF-1 upon activation but are indeed the major source of serum SDF-1. Therefore, we determined SDF-1 levels in a lysate prepared from platelet-rich plasma (PRP) containing a known number of platelets. We found an absolute SDF- 1 content of $2.04 \pm 0.33 \mathrm{pg} / 10^{6}$ TSP-DKO platelets and $1.55 \pm 0.41 \mathrm{pg} / 10^{6} \mathrm{WT}$ platelets $(n=6, P=0.16)$. Although this difference did not reach significance either, the combination of higher platelet levels and a higher platelet SDF-1 content resulted in a significantly higher quantity of SDF- $1 / \mathrm{ml}$ of whole blood in TSP-DKO as compared with WT mice. In fact, TSP-DKO mouse blood contained almost twice as much SDF-1 as WT mouse blood $(3.06 \pm 0.5 \mathrm{ng} / \mathrm{ml}$ versus $1.67 \pm 0.5 \mathrm{ng} / \mathrm{ml}, n=6, P=0.03)$. In order to prove that this increase in SDF-1 content was derived from the platelet releasate, we incubated WT as well as TSP-DKO platelets with ${ }^{14} \mathrm{C}$-labeled serotonin and activated them with different agonists. Results were monitored by platelet aggregometry, and serotonin release was measured. The highest percentage of serotonin was released upon stimulation with collagen $(10 \mu \mathrm{g} / \mathrm{ml})$ followed by stimulation with ADP $(10 \mu \mathrm{mol})$. Correlating SDF-1 release by the same platelet samples, we found that the largest quantity of SDF-1 was released from those platelets that released the maximal quantity of serotonin. As we expected from the above results, the releasates from TSP-DKO platelets contained about twice the absolute amount of SDF-1 per $10^{6}$ platelets as WT platelets (Figure 8C; blood from $n=3-4$ animals was pooled to prepare PRP, experiment repeated 3 times; $P<0.05)$. This difference was also observed upon stimulation with collagen only, albeit at lower absolute levels. The key finding in these experiments, however, was the fact that TSP-DKO platelets could differentially secrete higher proportions of their SDF-1 content as compared with their serotonin content. When the ratio of the secretion of SDF-1 to serotonin was calculated, TSP-DKO platelets consistently and differentially secreted a significantly higher percentage of SDF-1 (Table 1). This finding not only challenges the notion that platelets cannot differentially secrete their contents, but it also led us to ask the question of how the mere presence or absence of TSPs in platelets could influence SDF-1 secretion. Human platelets have been shown to contain MMP-1, MMP-2, MMP-3, and MMP-9 (54). We have demonstrated that MMP-9 is expressed and secreted by activated murine megakaryocytes (55). Reportedly, MMP-9 may function as an inhibitor of platelet aggregation while MMP-2 stimulates platelet aggregation (7). In addition, TSP1 is known to inhibit MMP-3 and MMP-9 zymogens in vitro (56) and in vivo (29). Therefore, one mechanism by which TSPs affected the secretion of SDF-1 from platelet stores may have been inhibition of proteases. We previously reported that platelets from MMP-9-/- mice display a defect in releasing SDF-1 after stimulation with thrombin (45). Therefore, we examined platelets derived from MMP-9-/- mice under the same conditions as WT and TSP-DKO platelets. Activation of MMP-9-/- platelets was intact but delayed as compared with that of WT platelets. Under the same conditions, MMP-9-/- platelets released less SDF-1 than WT platelets. These data suggest that TSP-dependent inhibition of MMP-9 (previously demonstrated in tumor models; ref. 29) controls SDF-1 release by platelets. However, the addition of exogenous TSP1 to TSP-DKO PRP was not sufficient to inhibit their increased release of SDF-1 (data not shown). This result furthermore stresses the importance of intracellular stores rather than secreted accumulations of TSPs in the regulation of thrombopoietic cell performance.

Importantly, we observed that MMP-9 activity in conditioned media from cultured megakaryocytes was substantially lower in media from WT as compared with TSP-DKO megakaryocytes, confirming previous observations (56) (data not shown).

SDF-1 expression in thrombopoietic cells. Increased SDF-1 secretion by platelets from TSP-DKO animals could be due to increased SDF-1 production in TSP-DKO megakaryocytes as compared with that in WT megakaryocytes. To test this hypothesis, we performed real-time quantitative PCR (qPCR) on lineage-negative precursors from both WT and TSP-DKO mice. SDF-1 mRNA abundance was normalized 

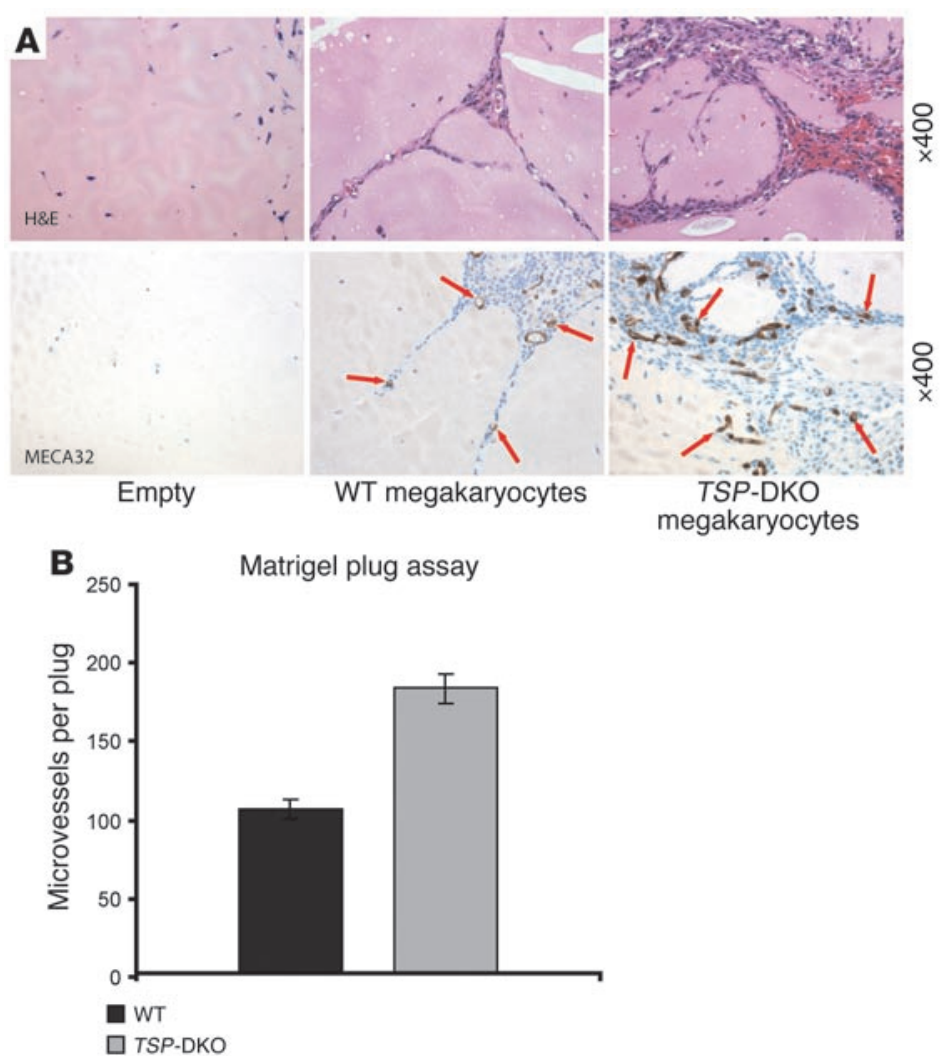

Figure 9

TSP-DKO megakaryocytes are highly angiogenic in a Matrigel plug assay (A) Megakaryocytes derived from TSP-DKO or WT mice were resuspended in growth factor-depleted Matrigel, injected subcutaneously, and plugs were harvested 3 weeks later. While the control plugs containing only carrier solution were virtually free of vasculature, the megakaryocyte-containing plugs showed vascular channel formation (red arrows) both in the WT and in the TSP-DKO megakaryocyte-loaded plugs. Representative fields were stained with H\&E and anti-panendothelial cell antigen marker clone MECA32 (blood vessels). Scored at original magnification, $\times 400$. (B) The number of MECA32+ neovessels was significantly higher in the plugs loaded with TSP-DKO megakaryocytes $(n=3, P<0.05)$.

to $\beta$-actin as endogenous control. When the same lineage-negative cells were differentiated into megakaryocytes in vitro using thrombopoietin as the single hematopoietic cytokine, SDF-1 mRNA was downregulated by about 1,000-fold within the first 4 days (Supplemental Figure 3). In the same WT cells, TSP1 mRNA was shown to increase approximately 4-fold during incubation while CXCR4 transcription remained stable. Therefore, the absence of TSPs does not result in increased transcription of the SDF-1 gene in megakaryocytes. In fact, SDF-1 mRNA is low in mature megakaryocytes. However, when human megakaryocytes were derived in vitro from $\mathrm{CD} 34^{+}$cord blood cells and subsequently stained for SDF-1, a granular staining within the cytoplasm could be identified (data not shown). In addition, staining of murine bone marrow for SDF-1 revealed that megakaryocytes contained SDF- 1 in situ. These findings are in line with a recent report in which SDF-1 was found to be localized in megakaryocytic $\alpha$-granules (57). However, our results suggest that, as with serotonin and fibrinogen, SDF-1 may be taken up into megakaryocyte secretory organelles rather than expressed by megakaryocytes.
Megakaryocytes induce angiogenesis in Matrigel plugs. In order to establish the angiogenic phenotype of WT as compared with TSP-DKO megakaryocytes, we examined vascular channel formation in cytokine-depleted Matrigel plugs. To this end, Matrigel plugs were loaded with 300,000 megakaryocytes obtained from WT or TSP-DKO mice and injected subcutaneously into the lateral abdominal wall region. After 21 days, the plugs were harvested and examined for microvascular density. Remarkably, megakaryocytes were found to induce extensive vascular channel formation, with neovessels containing erythrocytes in the case of both WT and TSP-DKO megakaryocytes whereas acellular control Matrigel plugs were devoid of any vascular channels (Figure 9A). Closer examination of H\&E-stained as well as MECA32-stained Matrigel plugs revealed that the vascular channels contained invading hematopoietic cells as well as abundant MECA $32^{+}$microvessels and that TSP-DKO megakaryocytes induced an approximately 1.8-fold higher vascularization of plugs as compared with WT megakaryocytes (Figure 9B). Megakaryocytes have been known to contain angiogenic factors, such as VEGF-A, and our data suggest that their proangiogenic effect is potentiated in the absence of TSPs. Therefore, TSPs within the platelets act as an angiogenic control system modulating the angiogenic performance of platelets.

\section{Discussion}

The mechanism by which thrombopoiesis regulates the angiogenic switch is not known. Here, we have demonstrated that the expression and release of TSPs stored in megakaryocytes and platelets confers an antiangiogenic effect. Although TSP1 is elaborated by megakaryocytes, TSP2 has been shown to be secreted by subsets of cultured marrow stromal cells and incorporated into megakaryocytes (58), modulating megakaryocytopoiesis (59). Here, we show that cellular TSP1 and TSP2 stored in megakaryocytes not only regulate thrombopoiesis but also revascularization of the myelosuppressed bone marrow and ischemic limbs. Thrombopoiesis is accelerated in mice deficient in TSP1 and TSP2, leading to rapid platelet recovery and enhanced assembly of blood vessels in the marrow and ischemic limbs. These data suggest that deployment of TSPs by thrombopoietic cells in the marrow or induced release by the platelets exerts a temporizing effect in regulating angiogenesis within the regenerating marrow and ischemic limbs.

Reciprocal interaction between bone marrow microvascular sinusoids and megakaryocytes is critical for both thrombocytopoiesis and marrow revascularization. Importantly, during recovery from myelosuppression, the absence of TSPs accounted for both a higher number of proliferating megakaryocytes and a higher quantity of sinusoidal neovessels. We demonstrate that inhibition of sinusoidal neoangiogenesis and remodeling following myelosuppression with the vascular targeting agent CA4P not only causes vascular disruption in the bone marrow but also inhibits megakaryocyte recovery. In addition, it markedly decreases the TSP-deficient thrombocytotic phenotype. In Matrigel angiogenic assays, TSP is the major determinant of the megakaryocyte angiogenic phenotype. Thus, TSP-deficient megakaryocytes manifest much more robust proangiogenic activity than WT cells. 
In parallel with bone marrow revascularization, peripheral microvascular beds in the process of vascular recovery after induction of ischemia are controlled by megakaryocyte-derived TSPs. In this instance, the megakaryocyte progeny in the form of platelets provide TSPs to perivascular areas, thereby limiting neoangiogenesis. Significantly, selective removal of TSP1 and TSP2 alone in $\mathrm{KO}$ mice results in substantial improvement in ischemic revascularization of the hind limb. Moreover, WT mice transplanted with TSP-DKO bone marrow displayed enhanced recovery from hind limb ischemia as compared with TSP-DKO mice transplanted with WT marrow. This finding provides further evidence that TSPs derived from transplantable cells not only negatively regulate marrow sinusoidal density but also moderate the rate and extent of revascularization of ischemic limbs. These data suggest that the absence of TSPs from thrombopoietic cells confers a proangiogenic phenotype to TSP-DKO mice as well as to WT recipients of TSP-DKO marrow.

In support of this concept, we demonstrate that transfusion of TSP-DKO mice with $3 \times 10^{8} \mathrm{WT}$ platelets was sufficient to promote selective, substantial deployment of TSPs around microvessels in the ischemic hind limb. However, this effect did not induce a significant inhibition of recovery from hind limb ischemia in these mice. The failure of TSP-DKO platelets to enhance recovery in WT animals may be related to the fact that these mice have normal platelet levels, resulting in WT microenvironmental conditions in ischemic tissue niches. Indeed, to highlight the importance of the platelet dose in mediating neoangiogenesis, Amano et al. reported proangiogenic activity by transfused WT platelets in thrombopoietindeficient $\left(\mathrm{Thpo}^{-/-}\right)$thrombocytopenic animals (8). In our studies, transfusion of $3 \times 10^{8}$ platelets elevated the platelet count of recipient animals by approximately $150-200 \times 10^{3} / \mu \mathrm{l}$ for $2-3$ days. However, this may not have resulted in sufficient platelet elevation to have an impact on the generation of a proangiogenic microenvironmental condition in ischemic tissue, where TSPs could have been deposited prior to the transfusion of TSP-DKO platelets.

The finding that Matrigel plugs loaded with either WT or TSP-DKO megakaryocytes were heavily infiltrated with neoangiogenic vessels confirms the net proangiogenic phenotype of thrombopoietic cells $(17,60)$. This underscores the importance of the absence of TSP1 and TSP2 in a complex mixture of pro- and antiangiogenic factors contained within thrombopoietic cells. However, the mere content of angiogenic factors, such as VEGF-A or SDF-1, may be less important functionally than the capacity of thrombopoietic cells to release these mediators. We demonstrate that SDF-1 is released from platelets in an activation-dependent manner. We correlated our findings with the demonstration of serotonin release in parallel with SDF-1 release. The increase in the release of serotonin with stronger agonists was paralleled at all times with increasing release of SDF-1. Of note, TSP-DKO platelets were found to release almost twice as much SDF-1 upon stimulation as WT platelets. However, in contrast, MMP-9-/platelets were more sensitive to stimulants and aggregated more readily but did not release substantial amounts of SDF-1. Since TSPs are potent inhibitors of MMP-9, this finding suggests that in the presence of TSPs, proteolytic activity under these microenvironmental conditions is inhibited. This may then limit release of SDF-1 from platelet stores.

We demonstrate that bone marrow megakaryocytes may be the most important hematopoietic cell type stabilizing the specialized, discontinuous bone marrow sinusoidal endothelium. We propose what we believe is a novel concept, that pro- and antiangiogenic proteins contained within both megakaryocytes and platelets determine the angiogenic phenotype of thrombopoietic cells. The proangiogenic phenotype of TSP-deficient thrombopoietic cells is quite evident in the bone marrow, where the absence of TSPs from megakaryocytes results in higher sinusoidal density. This also occurs in nonhematopoietic organs, where the absence of TSPs from platelets results in more rapid recovery from hind limb ischemia. We propose that the mechanism by which TSP deficiency confers a proangiogenic phenotype is through a reduction of inhibition of proteases, supporting the previous finding that platelets obtained from MMP-9-deficient mice have a defect in SDF-1 release (45).

The importance of SDF-1 in angiogenesis is now more fully appreciated. In addition, the major source of SDF-1 in ischemic areas is activated platelets. Thus, interference with SDF-1/ CXCR4 signaling may be an attractive target for antiangiogenic therapy. Moreover, our data identify 3 additional therapeutic targets: platelets, MMPs, and TSPs. Whereas all 3 have previously been implicated as key factors in angiogenesis, our results now provide a mechanistic link among these factors during marrow regeneration and revascularization of the ischemic limbs. Cellular TSPs in thrombopoietic cells are a major determinant of the angiogenic phenotype of megakaryocytes and their platelet progeny. This relates not only to megakaryocytes in the bone marrow but also to ischemic sites, where TSPs are deposited by activated platelets and where SDF-1, controlled by the proteolytic microenvironment, may be cleaved from the platelet surface. The proteolytic microenvironment, in turn, is influenced by the quantity of released TSPs. Thus, stored TSPs within the thrombopoietic cells may represent the major control pathway limiting excessive megakaryocyte and platelet-mediated promotion of angiogenesis. Delivery of hematopoietic cells depleted of TSPs may provide for an effective means of accelerating revascularization of regenerating tissue in preclinical and clinical settings.

\section{Methods}

Animals. The derivation of TSP1-deficient and TSP2-deficient mice has been described in detail elsewhere $(61,62)$. These mice were rederived on a 129/C57BL/ 6 background, and TSP-DKO mice were generated by crossbreeding $\mathrm{TSP}^{-/-}$and $\mathrm{TSP}^{-/-}$mice. Littermate mice were used as WT controls. All animals were maintained in air-filtered Thorensen units. Animal experiments were performed with the authorization of the institutional review board of the Animal Care and Use Committee of Weill Medical College of Cornell University. All experiments were performed using age- and sex-matched, 6-to 12-week-old animals.

Ischemic hind limb procedure. Groups of untreated mice or groups of mice 6 weeks after bone marrow transplantation underwent ligation and segmental resection of left femoral vessels. To induce hind limb ischemia, a midline incision of the abdominal skin was made, and extraperitoneal dissection laterally along the tissue planes was performed to expose the femoral vessels in the left inguinal area. The artery was then ligated both proximally and distally using 6-0 silk sutures, and the ligated vessels were resected between the ligatures without damaging the femoral nerve. The midline incision was then closed using 4-0 POLYSORB sutures (United States Surgical). Rate of blood flow was measured every 3 days after ligation by means of a laser Doppler perfusion imaging system (Lisca PIM II Laser Doppler; Perimed) in both the ischemic and nonischemic hind limbs of the same animal. The ratio of relative blood flow was then computed by dividing the laser Doppler-derived blood flow of the ischemic hind limb 
by that of the nonischemic hind limb and plotting over time. At 28 days, the mice were sacrificed by $\mathrm{CO}_{2}$ inhalation, and tissues were harvested for hind limb skeletal muscle histology.

Immunohistochemistry of the skeletal muscle in hind limb ischemia. Hind limb gastrocnemii were dissected and either snap-frozen in liquid nitrogen or, alternatively, fixed in $4 \%$ paraformaldehyde (PFA). PFA-fixed tissue was either embedded in paraffin or snap-frozen in OCT. Fixed paraffin or cryosections $(5-8 \mu \mathrm{m})$ were stained with rat anti-mouse CD31 (PECAM-1; BD Biosciences - Pharmingen) followed by donkey anti-rat IgG Cy3 (Jackson ImmunoResearch Laboratories Inc.) or alternatively with mouse biotinylated anti-TSP1 antibody (TSP Ab-4) (Lab Vision Corp.) followed by streptavidin-HRP (Jackson ImmunoResearch Laboratories Inc.) or streptavidin-Cy2 (Jackson ImmunoResearch Laboratories Inc.). All chromogen developed was done with $\mathrm{DAB}+(\mathrm{DAB}$, diaminobenzidine) per manufacturer's instructions (Dako). Fresh-frozen sections of hind limb gastrocnemii or adductor muscles were stained with rat anti-mouse CD41 ( $\alpha$-integrin GpIIb; BD Biosciences - Pharmingen) followed by biotinylated donkey anti-rat IgG and streptavidin-HRP with DAB+ development as above. Quantitation of immunohistochemical staining was performed with a minimum of 5 regions of the skeletal muscle for 3-5 animals per condition; samples were photographed and images were subjected to NIH Image analysis by investigators blinded to experimental conditions.

Myelosuppression model. For myelosuppression experiments, mice received a single tail-vein injection of $5-\mathrm{FU}(250 \mathrm{mg} / \mathrm{kg}$ body weight).

Total-body irradiation and bone marrow transplantation. Experimental mice were irradiated with a dose of $9.5 \mathrm{~Gy}$ from a ${ }^{137} \mathrm{Cs}$-ray source at a dose rate of approximately $0.90 \mathrm{~Gy} / \mathrm{min}$. Immediately after lethal irradiation, total bone marrow cells from donor animal femurs were injected into the tail vein.

Peripheral-blood analysis. Retro-orbital blood was collected on indicated days with capillary pipettes (Fisher Scientific). Differential blood counts were obtained using an automated Bayer ADVIA 120 MultiSpecies Hematology Analyzer (Bayer HealthCare). Peripheral blood counts are reported as average values \pm SEM.

SDF-1 ELISA. SDF-1 levels were measured in serum, platelet-poor plasma, platelet lysate (PRP in $0.01 \%$ Triton $\mathrm{X}$ ), and platelet releasate using an SDF-1 ELISA kit according to the instructions of the manufacturer (Quantikine Immunoassays; R\&D Systems).

Bone marrow histology. Femurs of experimental mice were harvested, washed in ice-cold PBS, and fixed in $4 \%$ PFA at $4{ }^{\circ} \mathrm{C}$ for 4 hours. After decalcification, tissue was embedded in paraffin and sectioned $(5 \mu \mathrm{m})$. For immunohistochemistry, slides were deparaffinized and rehydrated. After heat-induced antigen retrieval, steps were taken to block endogenous peroxidase activity and nonspecific antibody binding. Slides were then incubated with monoclonal rat anti-mouse panendothelial cell antigen (MECA-32; BD Biosciences - Pharmingen), polyclonal rabbit anti-human citrulline (07-377; Upstate USA Inc.), or biotinylated monoclonal mouse anti-human TSP1 (TSP Ab-4; Lab Vision Corp.). Where necessary, biotinylated donkey anti-rat IgG and donkey anti-rabbit IgG secondary antibodies (Jackson ImmunoResearch Laboratories Inc.) were employed. All staining was developed with $\mathrm{DAB}+$ (Dako). Sections were counterstained with Mayer's hematoxylin. Megakaryocytes were counted in spleen and bone marrow as previously described (63).

Bone marrow cytospin immunofluorescence. Femurs were harvested from WT mice and flushed, and cytospin preparations were made from marrow single-cell suspension. Slides were dried, fixed in 10\% neutral buffered formaline for 10 minutes, and subsequently blocked in 5\% BSA. Cytospins were incubated with rabbit anti-citrulline antibody (07-377; Upstate USA Inc.) and visualized using an anti-rabbit IgG Cy2- or Cy3-coupled secondary antibody (Jackson ImmunoResearch Laboratories Inc.). In addition, for direct visualization of antibody binding to the respective target cells, each slide was incubated with 1 of the following phycoerythrin-coupled, lineage-specific antibodies: anti-CD11b, anti-CD45R, anti-Gr-1, anti-Ter119, anti-CD3, and anti-CD41a (all from BD Biosciences - Pharmingen).

Histologic image acquisition and analysis. Images of bone marrow sections were taken with a digital camera (Retigo EX; QImaging) mounted on an Olympus BX51 microscope (Olympus). UPLFLN 10×/0.25, 20×/0.50, and $40 \times / 0.75$ objective lenses were used. Images were recorded using Mac QCapture Acquisition software (version 2.68.6; QImaging).

Digital recordings of the tissue slides were analyzed for differential chromogen content. In brief, Adobe Photoshop software (version 7.0; Adobe) was used for color selection. Using Photoshop-based image analysis as described previously (64), we quantified the brown chromogen by counting the number of brown pixels as a measure of spatial distribution.

Mouse platelet aggregation and release assays. Mice were anesthetized and, using a short length $(\sim 1 \mathrm{~cm})$ of heparinized hematocrit tubing, we collected $750 \mu \mathrm{l} \mathrm{blood}$ from the retrobulbar venous plexus, transferring it directly into a mixture of $675 \mu \mathrm{l}$ saline $(165 \mathrm{mM} \mathrm{NaCl})$ and $75 \mu \mathrm{l} \mathrm{3.2 \%}$ sodium citrate. Blood from 3-4 mice was pooled and centrifuged $\left(120 \mathrm{~g}, 5\right.$ minutes, $\left.25^{\circ} \mathrm{C}\right)$ to obtain PRP $(65,66)$. In order to maximize the yield of platelets, the blood remaining from this first centrifugation was diluted with mouse saline to the original volume, gently mixed, and centrifuged as before. Thus, the resulting pooled PRP was diluted approximately 4-fold; this was confirmed by a complete blood count of the PRP (ADVIA 120 Automated Hematology Analyzer; Bayer HealthCare). Platelet counts in PRP were consistently approximately $400,000 / \mu l$. Platelet dense bodies were labeled by incubation of PRP with $\left[{ }^{14} \mathrm{C}\right]-5$-hydroxytryptamine $\left(\left[{ }^{14} \mathrm{C}\right]-5 \mathrm{HT}\right.$, also known as serotonin, $57.5 \mathrm{mCi} / \mathrm{mmol}, 0.2 \mathrm{nmol} / \mathrm{ml} \mathrm{PRP}, 30$ minutes, $\left.25^{\circ} \mathrm{C}\right)$. Aggregometry, using platelet-poor plasma as reference, was performed using a ChronoLog model 490 aggregometer system $(65,67)$. Agonists tested (Chrono-Log) included ADP $(10$ and $5 \mu \mathrm{M})$ and collagen (10 and $5 \mu \mathrm{g} / \mathrm{ml})$. PRP was preincubated with $2.5 \mu \mathrm{M}$ imipramine for 3 minutes before agonist addition in order to prevent platelet reuptake of released $5 \mathrm{HT}$. Controls containing labeled platelets without agonist were carried out to measure any release of $\left[{ }^{14} \mathrm{C}\right]-5 \mathrm{HT}$ attributable to stirring alone; these blanks were subtracted from both the total counts in PRP and the counts measured in cell-free releasates. Reactions were stopped 5 minutes $(7$ minutes when using collagen) after agonist addition by placing the cuvettes on ice. Cuvette contents were transferred to microfuge tubes and centrifuged $(1$ minute, $14,000 \mathrm{~g})$. Then, $50 \mu \mathrm{l}$ aliquots of each supernatant were counted in $4 \mathrm{ml}$ scintillation liquid. Release of $5 \mathrm{HT}$ was expressed as the percentage [(counts in supernatants - counts in blank)/(counts in PRP - counts in blank)] indicated in the figure(s). Extent of aggregation was expressed as area under the curve (arbitrary units), as determined by AGGRO/LINK software (version 5.2.2; Chrono-Log). Release of SDF-1 was expressed as percentage of total (lysed PRP or serum) or as $\mathrm{pg} / 10^{6}$ platelets in each test.

RNA extraction and reverse transcription. RNA from megakaryocytes was extracted using the RNAqueous-Micro kit (Ambion) according to manufacturer's instructions, including DNase I treatment. Superscript II (Invitrogen) was used according to the manufacturer's instructions for cDNA synthesis. qPCR. qPCR was performed using a 7500 Fast Real-Time PCR System (Applied Biosystems) in standard mode with Power SYBR Green (Applied Biosystems). The qPCR conditions were as follows: $50^{\circ} \mathrm{C}$ for 2 minutes and $95^{\circ} \mathrm{C}$ for 10 minutes, followed by 40 cycles of $95^{\circ} \mathrm{C}$ for 15 seconds and $60^{\circ} \mathrm{C}$ for 1 minute. The following primers (except for SDF-1) were designed using Primer Express Software, version 3.0 (Applied Biosystems), and custom synthesized by Invitrogen: $\beta$-actin, forward 5 '-CTGCTCTGGCTCCTAGCACC-3', reverse 5'-CGCTCAGGAGGAGCAATGA-3'; TSP1, forward 5'-CGAGACAACTGCCAGTACGTT-3', reverse 5'-GTTCTGATGGCCATCCTCATC-3'; and SDF-1 $\alpha$, forward 5'-GAGCCAACGTCAAGCATCTG-3', reverse 5'-CGGGTCAATGCACACTTGTC-3' (68). 
Data were analyzed using SDS software, version 1.3.1 (Applied Biosystems). The $\Delta$ threshold cycle (Ct) method was used to obtain relative quantification; i.e., Ct values of the target genes (TSP1 and SDF-1) were normalized to the corresponding $\mathrm{Ct}$ value of the control gene ( $\beta$-actin). Relative expression was calculated as follows: relative expression $=(2 \Delta \mathrm{Ct}) \times 10,000$. No-template controls and minus RT controls were run as appropriate. Each qPCR was followed by a dissociation curve analysis to confirm the specific amplification of the target.

In vivo Matrigel studies. Bone marrow was harvested from WT and TSP-DKO mice, and lineage negative cells were selected using the Miltenyi Lineage Negative Selection kit according to the manufacturer's instructions (Miltenyi Biotec). Cells were cultured for 4 days in X-VIVO and $10 \%$ fetal bovine serum supplemented with $50 \mathrm{ng} / \mathrm{ml}$ thrombopoietin. More than $90 \%$ of the resulting cells were CD $41^{+}$megakaryocytes. Then, $300,000 \mathrm{WT}$ or TSP-DKO megakaryocytes were harvested and suspended in growth factor-depleted Matrigel (BD; $300 \mu \mathrm{l})$ with heparin $(20 \mathrm{U} / \mathrm{ml})$. Matrigel with heparin alone was used as a control. The Matrigel plugs were placed subcutaneously in the abdominal regions of WT mice. Mice were sacrificed after 3 weeks, and the Matrigel plugs were dissected and fixed for 4 hours at $4{ }^{\circ} \mathrm{C}$ in $4 \%$ PFA, followed by dehydration and paraffin embedding. For histological analysis, paraffin-embedded sections were processed and stained using H\&E. Immunohistochemical analysis was performed using MECA32 antibody as described under "Bone marrow histology." Quantification of vessel density was performed on 6 central sections for each sample, using a gridded eyepiece, by investigators blinded to sample conditions. The total number of MECA32 ${ }^{+}$vessels visible per plug was counted.
Statistics. Results were statistically analyzed using a 2-tailed Student's $t$ test. Results are expressed as mean value \pm SEM. A $P$ value of less than 0.05 was considered significant.

\section{Acknowledgments}

We thank Jack Lawler for providing expertise and thrombospondindeficient mice. S. Rafii was supported by the Howard Hughes Medical Institute, the NIH (grants R01 HL075234, R01 HL59312, R01 HL67839, R01 HL61849, and R21 HL083222), the American Cancer Society, and the Leukemia and Lymphoma Society. This study was also supported by grants from the Mildred Scheel Stiftung, Deutsche Krebshilfe, Bonn, Germany (to H.-G. Kopp and T. Milde) and the NIH (R37 HL47073, P01 HL46403, and R01 NS41462 to A.J. Marcus and M.J. Broekman) as well as Merit Review grants from the Department of Veterans Affairs (to A.J. Marcus and M.J. Broekman). P. Bornstein was supported by NIH grant R01 AR45418. We acknowledge the excellent technical assistance of Ali M. Farooki.

Received for publication June 6, 2006, and accepted in revised form October 24, 2006.

Address correspondence to: Shahin Rafii, Howard Hughes Medical Institute, Weill Medical College of Cornell University, 1300 York Avenue, Room A-863, New York, New York 10021, USA. Phone: (212) 746-2070; Fax: (212) 746-8481; E-mail: srafii@med.cornell.edu.
1. Billroth, T. 1878. Lectures on surgical pathology and therapeutics. A handbook for students and practitioners. New Syndenham Society. London, United Kingdom. 355 pp.

2. Honn, K.V., Tang, D.G., and Crissman, J.D. 1992. Platelets and cancer metastasis: a causal relationship? Cancer Metastasis Rev. 11:325-351.

3. Sierko, E., and Wojtukiewicz, M.Z. 2004. Platelets and angiogenesis in malignancy. Semin. Thromb. Hemost. 30:95-108.

4. Rhee, J.S., et al. 2004. The functional role of blood platelet components in angiogenesis. Thromb. Haemost. 92:394-402.

5. Mohle, R., Green, D., Moore, M.A., Nachman, R.L., and Rafii, S. 1997. Constitutive production and thrombin-induced release of vascular endothelial growth factor by human megakaryocytes and platelets. Proc. Natl. Acad. Sci. U. S. A. 94:663-668.

6. Pintucci, G., et al. 2002. Trophic effects of platelets on cultured endothelial cells are mediated by platelet-associated fibroblast growth factor-2 (FGF-2) and vascular endothelial growth factor (VEGF). Thromb. Haemost. 88:834-842.

7. Fernandez-Patron, C., et al. 1999. Differential regulation of platelet aggregation by matrix metalloproteinases-9 and -2. Thromb. Haemost. 82:1730-1735.

8. Amano, H., Hackett, N.R., Rafii, S., and Crystal, R.G. 2005. Thrombopoietin gene transfer-mediated enhancement of angiogenic responses to acute ischemia. Circ. Res. 97:337-345.

9. Verheul, H.M., et al. 1997. Platelet: transporter of vascular endothelial growth factor. Clin. Cancer Res. 3:2187-2190.

10. English, D., et al. 2000. Sphingosine 1-phosphate released from platelets during clotting accounts for the potent endothelial cell chemotactic activity of blood serum and provides a novel link between hemostasis and angiogenesis. FASEB J. 14:2255-2265.

11. English, D., Garcia, J.G., and Brindley, D.N. 2001. Platelet-released phospholipids link haemostasis and angiogenesis. Cardiovasc. Res. 49:588-599.

12. Pipili-Synetos, E., Papadimitriou, E., and Maragoudakis, M.E. 1998. Evidence that platelets promote tube formation by endothelial cells on matrigel.

\section{Br. J. Pharmacol. 125:1252-1257.}

13. Gasic, G.J., Gasic, T.B., and Stewart, C.C. 1968. Antimetastatic effects associated with platelet reduction. Proc. Natl. Acad. Sci. U. S. A. 61:46-52.

14. Amirkhosravi, A., et al. 2003. Inhibition of tumor cell-induced platelet aggregation and lung metastasis by the oral GpIIb/IIIa antagonist XV454. Thromb. Haemost. 90:549-554.

15. Varner, J.A., Nakada, M.T., Jordan, R.E., and Coller, B.S. 1999. Inhibition of angiogenesis and tumor growth by murine 7E3, the parent antibody of c7E3 Fab (abciximab; ReoPro). Angiogenesis. 3:53-60.

16. Falanga, A., and Piccioli, A. 2005. Effect of anticoagulant drugs in cancer. Curr. Opin. Pulm. Med. 11:403-407.

17. Kisucka, J., et al. 2006. Platelets and platelet adhesion support angiogenesis while preventing excessive hemorrhage. Proc. Natl. Acad. Sci.U. S. A. 103:855-860.

18. Maione, T.E., et al. 1990. Inhibition of angiogenesis by recombinant human platelet factor-4 and related peptides. Science. 247:77-79.

19. Iruela-Arispe, M.L., Bornstein, P., and Sage, H. 1991. Thrombospondin exerts an antiangiogenic effect on cord formation by endothelial cells in vitro. Proc. Natl. Acad. Sci. U. S. A. 88:5026-5030.

20. Baenziger, N.L., Brodie, G.N., and Majerus, P.W. 1971. A thrombin-sensitive protein of human platelet membranes. Proc. Natl. Acad. Sci. U. S. A. 68:240-243.

21. Lawler, J. 1986. The structural and functional properties of thrombospondin. Blood. 67:1197-1209.

22. Good, D.J., et al. 1990. A tumor suppressor-dependent inhibitor of angiogenesis is immunologically and functionally indistinguishable from a fragment of thrombospondin. Proc. Natl. Acad. Sci. U. S. A. 87:6624-6628

23. Bornstein, P., Devarayalu, S., Li, P., Disteche, C.M., and Framson, P. 1991. A second thrombospondin gene in the mouse is similar in organization to thrombospondin 1 but does not respond to serum. Proc. Natl. Acad. Sci. U. S. A. 88:8636-8640.

24. Adams,J.C., and Lawler,J.2004. The thrombospondins. Int. J. Biochem. Cell Biol. 36:961-968.

25. Bornstein, P. 2001. Thrombospondins as matricellular modulators of cell function. J. Clin. Invest.
107:929-934.

26. Chen, H., Herndon, M.E., and Lawler, J. 2000. The cell biology of thrombospondin-1. Matrix Biol. 19:597-614.

27. Lawler, J. 2002. Thrombospondin-1 as an endogenous inhibitor of angiogenesis and tumor growth. J. Cell. Mol. Med. 6:1-12.

28. Murphy-Ullrich, J.E., and Poczatek, M. 2000. Activation of latent TGF-beta by thrombospondin-1: mechanisms and physiology. Cytokine Growth Factor Rev. 11:59-69.

29. Rodriguez-Manzaneque, J.C., et al. 2001. Thrombospondin-1 suppresses spontaneous tumor growth and inhibits activation of matrix metalloproteinase- 9 and mobilization of vascular endothelial growth factor. Proc. Natl. Acad. Sci.U.S. A. 98: $12485-12490$.

30. Yao, L., et al. 2000. Thrombospondin-1 expression in oral squamous cell carcinomas: correlations with tumor vascularity, clinicopathological features and survival. Oral Oncol. 36:539-544.

31. Bleuel, K., Popp, S., Fusenig, N.E., Stanbridge, E.J., and Boukamp, P. 1999. Tumor suppression in human skin carcinoma cells by chromosome 15 transfer or thrombospondin-1 overexpression through halted tumor vascularization. Proc. Natl. Acad. Sci. U. S. A. 96:2065-2070.

32. Clezardin, P., Frappart, L., Clerget, M., Pechoux, C. and Delmas, P.D. 1993. Expression of thrombospondin (TSP1) and its receptors (CD36 and CD51) in normal, hyperplastic, and neoplastic human breast. Cancer Res. 53:1421-1430.

33. Coppinger, J.A., et al. 2004. Characterization of the proteins released from activated platelets leads to localization of novel platelet proteins in human atherosclerotic lesions. Blood. 103:2096-2104.

34. Ishida-Yamamoto, A., et al. 2000. Decreased deiminated keratin $\mathrm{K} 1$ in psoriatic hyperproliferative epidermis. J. Invest. Dermatol. 114:701-705.

35. Kopp, H.G., et al. 2005. Tie2 activation contributes to hemangiogenic regeneration after myelosuppression. Blood. 106:505-513.

36. Chen, Y.Z., et al. 1997. Thrombospondin, a negative modulator of megakaryocytopoiesis. J. Lab. Clin. Med. 129:231-238. 
37. Avecilla, S.T., et al. 2004. Chemokine-mediated interaction of hematopoietic progenitors with the bone marrow vascular niche is required for thrombopoiesis. Nat. Med. 10:64-71.

38. Radley, J.M., Hodgson, G.S., and Levin, J. 1980. Platelet production after administration of antiplatelet serum and 5-fluorouracil. Blood. 55:164-166.

39. Chenaille, P.J., Steward, S.A., Ashmun, R.A., and Jackson, C.W. 1990. Prolonged thrombocytosis in mice after 5-fluorouracil results from failure to down-regulate megakaryocyte concentration. An experimental model that dissociates regulation of megakaryocyte size and DNA content from megakaryocyte concentration. Blood. 76:508-515.

40. Ebbe, S., Yee, T., and Phalen, E. 1989. 5-fluorouracil-induced thrombocytosis in mice is independent of the spleen and can be partially reproduced by repeated doses of cytosine arabinoside. Exp. Hematol. 17:822-826

41. Kopp, H.G., Avecilla, S.T., Hooper, A.T., and Rafii, S. 2005. The bone marrow vascular niche: home of HSC differentiation and mobilization. Physiology (Bethesda). 20:349-356.

42. Han, Z.C., and Caen, J.P. 1993. Are megakaryocytes and endothelial cells sisters? J. Lab. Clin. Med. 121:821-825.

43. Fliedner, T.M., Graessle, D., Paulsen, C., and Reimers, K. 2002. Structure and function of bone marrow hemopoiesis: mechanisms of response to ionizing radiation exposure. Cancer Biother. Radiopharm. 17:405-426.

44. Vincent, L., et al. 2005. Combretastatin A4 phosphate induces rapid regression of tumor neovessels and growth through interference with vascular endothelial-cadherin signaling. J. Clin. Invest. 115:2992-3006. doi:10.1172/JCI24586.

45. Jin, D.K., et al. 2006. Cytokine-mediated deployment of SDF-1 induces revascularization through recruitment of CXCR4(+) hemangiocytes. Nat. Med. 12:557-567.

46. Bornstein, P., Agah, A., and Kyriakides, T.R. 2004 The role of thrombospondins 1 and 2 in the regulation of cell-matrix interactions, collagen fibril formation, and the response to injury. Int. J. Biochem. Cell Biol. 36:1115-1125.
47. Shaw, T., Chesterman, C.N., and Morgan, F.J. 1984. In vitro synthesis of low molecular weight proteins in human platelets: absence of labelled release products. Thromb. Res. 36:619-631.

48. McRedmond, J.P., et al. 2004. Integration of proteomics and genomics in platelets: a profile of platelet proteins and platelet-specific genes. $\mathrm{Mol}$. Cell. Proteomics. 3:133-144.

49. Kieffer, N., Guichard, J., Farcet, J.P., Vainchenker, W., and Breton-Gorius, J. 1987. Biosynthesis of major platelet proteins in human blood platelets. Eur. J. Biochem. 164:189-195.

50. Jackson, K.A., Snyder, D.S., and Goodell, M.A. 2004. Skeletal muscle fiber-specific green autofluorescence: potential for stem cell engraftment artifacts. Stem Cells. 22:180-187.

51. Yamaguchi, J., et al. 2003. Stromal cell-derived factor-1 effects on ex vivo expanded endothelial progenitor cell recruitment for ischemic neovascularization. Circulation. 107:1322-1328.

52. Salcedo, R., et al. 1999. Vascular endothelial growth factor and basic fibroblast growth factor induce expression of CXCR4 on human endothelial cells: in vivo neovascularization induced by stromal-derived factor-1alpha. Am. J. Pathol. 154:1125-1135.

53. Grunewald, M., et al. 2006. VEGF-induced adult neovascularization: recruitment, retention, and role of accessory cells. Cell. 124:175-189.

54. Jurasz, P., Chung, A.W., Radomski, A., and Radomski, M.W. 2002. Nonremodeling properties of matrix metalloproteinases: the platelet connection. Circ. Res. 90:1041-1043.

55. Lane, W.J., et al. 2000. Stromal-derived factor 1induced megakaryocyte migration and platelet production is dependent on matrix metalloproteinases. Blood. 96:4152-4159.

56. Bein, K., and Simons, M. 2000. Thrombospondin type 1 repeats interact with matrix metalloproteinase 2. Regulation of metalloproteinase activity. J. Biol. Chem. 275:32167-32173.

57. Massberg, S., et al. 2006. Platelets secrete stromal cell-derived factor 1alpha and recruit bone marrowderived progenitor cells to arterial thrombi in vivo. J. Exp. Med. 203:1221-1233.

58. Hankenson, K.D., and Bornstein, P. 2002.
The secreted protein thrombospondin 2 is an autocrine inhibitor of marrow stromal cell proliferation. J. Bone Miner. Res. 17:415-425.

59. Kyriakides, T.R., et al. 2003. Megakaryocytes require thrombospondin-2 for normal platelet formation and function. Blood. 101:3915-3923.

60. Brill, A., Dashevsky, O., Rivo, J., Gozal, Y., and Varon, D. 2005. Platelet-derived microparticles induce angiogenesis and stimulate post-ischemic revascularization. Cardiovasc. Res. 67:30-38.

61. Lawler, J., et al. 1998. Thrombospondin-1 is required for normal murine pulmonary homeostasis and its absence causes pneumonia. J. Clin. Invest. 101:982-992.

62. Kyriakides, T.R., et al. 1998. Mice that lack thrombospondin 2 display connective tissue abnormalities that are associated with disordered collagen fibrillogenesis, an increased vascular density, and a bleeding diathesis. J. Cell Biol. 140:419-430.

63. Slayton, W.B., et al. 2002. The spleen is a major site of megakaryopoiesis following transplantation of murine hematopoietic stem cells. Blood. 100:3975-3982.

64. Lehr, H.A., van der Loos, C.M., Teeling, P., and Gown, A.M. 1999. Complete chromogen separation and analysis in double immunohistochemical stains using Photoshop-based image analysis. J. Histochem. Cytochem. 47:119-126.

65. Marcus, A.J., et al. 1991. Inhibition of platelet function by an aspirin-insensitive endothelial cell ADPase. Thromboregulation by endothelial cells. J. Clin. Invest. 88:1690-1696.

66. Santos, M.T., et al. 1991. Enhancement of platelet reactivity and modulation of eicosanoid production by intact erythrocytes. A new approach to platelet activation and recruitment. J. Clin. Invest. 87:571-580.

67. Drosopoulos, J.H., et al. 2000. Site-directed mutagenesis of human endothelial cell ecto-ADPase/ soluble CD39: requirement of glutamate 174 and serine 218 for enzyme activity and inhibition of platelet recruitment. Biochemistry. 39:6936-6943.

68. Semerad, C.L., et al. 2005. G-CSF potently inhibits osteoblast activity and CXCL12 mRNA expression in the bone marrow. Blood. 106:3020-3027. 